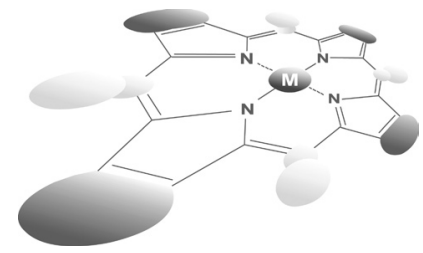

\title{
Room temperature ionic liquids based on cationic porphyrin derivatives and tetrakis(pentafluorophenyl)borate anion
}

\author{
Hai-Jun Xua, Claude P. Gros ${ }^{a \triangleleft}$, Stéphane Brandès ${ }^{a}$, Pei-Yu Ge ${ }^{b}$, Hubert H. Girault ${ }^{\mathrm{b} \diamond}$ \\ and Jean-Michel Barbe ${ }^{\star a} \diamond$
}

${ }^{a}$ Université de Bourgogne, ICMUB (UMR 5260), 9, Avenue Alain Savary, BP 47870, 21078 Dijon Cedex, France

${ }^{\mathrm{b}}$ Laboratoire d'Electrochimie Physique et Analytique, Station 6, Ecole Polytechnique Fédérale de Lausanne, Station 6, CH-1015 Lausanne, Switzerland

Dedicated to Professor Karl M. Kadish on the occasion of his $65^{\text {th }}$ birthday

Received 15 April 2011

Accepted 16 May 2011

\begin{abstract}
A series of 11 low melting ionic liquids based on meso-substituted $\mathrm{A}_{3} \mathrm{~B}$-porphyrins and $\mathrm{A}_{2} \mathrm{~B}_{2}$-porphyrins containing one or two pyridyl substituents have been synthesized in high yields. Three of them are liquids at room temperature. All these porphyrinic salts were characterized by ${ }^{1} \mathrm{H}$ NMR, ${ }^{19} \mathrm{~F}$ NMR, MALDI-TOF mass spectrometry, elemental analysis and UV-visible spectroscopy. The thermal properties and conductivity values of these salt derivatives have been also measured. A specific conductivity value of up to $4 \mathrm{mS} . \mathrm{cm}^{-1}$ could be obtained for a compound having the counter-anion $\mathrm{B}\left(\mathrm{C}_{6} \mathrm{~F}_{5}\right)_{4}$.
\end{abstract}

KEYWORDS: RT ionic liquids, $\mathrm{A}_{3} \mathrm{~B}$-porphyrins, $\mathrm{A}_{2} \mathrm{~B}_{2}$-porphyrins, conductivity, tetrakis(pentafluorophenyl)borate anion, DSC, TGA.

\section{INTRODUCTION}

Ionic liquids (ILs) are a group of organic salts that possess low melting point and usually afford liquids at temperatures below $100{ }^{\circ} \mathrm{C}$ [1]. Generally, ionic liquids consist completely of an organic cation and an anion (inorganic or organic). The ionic liquids which are free-flowing liquids at room temperature, can be called Room Temperature Ionic Liquids (RTILs) [1]. In recent years, ionic liquids have attracted much attention in many fields due to their unique chemical and physical properties such as air and moisture stability, high thermal stability, good electrical conductivity, high solubility, and no vapor pressure [1-9]. The ILs have been successfully used in many applications, including replacing traditional organic solvents in organic and inorganic syntheses [10], liquid-liquid

\footnotetext{
${ }^{\diamond}$ SPP full member in good standing

*Correspondence to: Jean-Michel Barbe, email: jean-michel. barbe@u-bourgogne.fr, tel: +33 (0)3-80-39-61-19, fax: +33 (0)3-80-39-61-17
}

extractions [11, 12], catalysis, electrochemical reactions $[13,14]$ as well as green solvents for lithium ion batteries [15-18] and for various reactions [19-21]. Up to now, most of the reported ILs are mainly based on 1,3-dialkylimidazolium cations, 1-alkylpyridinium cations and quaternary ammonium salts, whereas anions are mainly focused on $\left[\mathrm{BF}_{4}\right]^{-},\left[\mathrm{PF}_{6}\right]^{-},\left[\mathrm{CF}_{3} \mathrm{SO}_{3}\right]^{-},\left[\left(\mathrm{CF}_{3} \mathrm{SO}_{2}\right)_{2} \mathrm{~N}\right]^{-}$and $\left[\mathrm{RCO}_{2}\right]^{-}[1,22-26]$. However, there are only few examples where tetrakis(pentafluorophenyl)borate anion has been used in ILs [27], despite the fact that it can decrease the melting point of the salts due to its weak anion coordination properties, bulkiness and benefit to a good charge distribution [1].

In addition, porphyrins have also aroused remarkable and extensive interest for many years due to their wideranging applications in photophysics [28], coordination chemistry [29], liquid crystals [30], solar cells [31, 32], catalysis [33, 34], photovoltaic devices [35], photodynamic therapy (PDT) [36] and artificial light-harvesting antennas [37]. The excellent properties of ionic liquids and porphyrins stimulated us to develop ionic liquid 
porphyrin derivatives based on tetrakis(perfluorophenyl)borate salts and cationic porphyrins. The room temperature liquid state was attained by introducing long alkoxy chains on the meso phenyl groups of the porphyrins. During the progress of our work, Gryko and coworkers reported the synthesis of meso-substituted liquid porphyrins [38]. Concomitantly, Maruyama and coworkers independently reported the synthesis of room temperature liquid porphyrins [39]. Here, we report the first trial way to synthesize one of the most versatile ionic liquids. These ionic liquid porphyrins (Chart 1) have been examined by ${ }^{1} \mathrm{H}$ and ${ }^{19} \mathrm{~F}$ NMR, MALDI-TOF mass spectrometry, elemental analysis, DSC, TGA and UV-visible spectroscopy. Room temperature conductivity measurements have also been performed.

\section{EXPERIMENTAL}

\section{Instrumentation}

${ }^{1} \mathrm{H}$ and ${ }^{19} \mathrm{~F}$ NMR spectra were recorded with a Bruker DRX-300 AVANCE transform spectrometer at the "Plateforme d'Analyse Chimique de l'Université de Bourgogne" (PACSMUB); chemical shifts are expressed in $\mathrm{ppm}$ relative to chloroform. UV-vis spectra were recorded with a Varian Cary 1 spectrophotometer. Mass spectra and accurate mass measurements (HRMS) were obtained with a Bruker Daltonics Ultraflex II spectrometer in the MALDI/TOF reflectron mode by using dithranol as a matrix. Accurate mass measurements (HRMS) were carried out under the same conditions as before by using the PEG-ion series as an internal calibrant. Both measurements were made at the PACSMUB.

Thermogravimetric (TGA) measurements were performed on a Netzsch STA 409 PC Luxx analyzer at the ICMUB Institute. Samples were purged in an $\operatorname{Ar}(30$ $\left.\mathrm{mL} \cdot \mathrm{min}^{-1}\right) / \mathrm{O}_{2}\left(10 \mathrm{~mL} \cdot \mathrm{min}^{-1}\right)$ stream during analysis and heated to $900{ }^{\circ} \mathrm{C}$ in alumina crucibles with a heating rate of $10 \mathrm{~K} \cdot \mathrm{min}^{-1}$. Differential Scanning Calorimetry (DSC) measurements were performed on a Perkin Elmer Diamond DSC instrument. The DSC measurements were carried out under nitrogen at atmospheric pressure in a sealed $\mathrm{Al}$ pan with a heating rate of $5 \mathrm{~K} / \mathrm{min}$ or $10 \mathrm{~K} / \mathrm{min}$ at the "Ecole Polytechnique Fédérale de Lausanne, EPFL". Resistance of ionic liquid porphyrin was detected by an on-chip conductivity sensor connected to an AutoLab PGSTAT 12 potentiostat at EPFL.

\section{Chemicals and reagents}

Unless otherwise noted, all chemicals and solvents were of analytical reagent grade and used as received. Absolute dichloromethane $\left(\mathrm{CH}_{2} \mathrm{Cl}_{2}\right)$ was obtained from Carlo Erba. Silica gel (Merck; 70-120 mm) was used for column chromatography. Analytical thin-layer chromatography was performed with Merck 60 F254 silica gel (precoated sheets, $0.2 \mathrm{~mm}$ thick). Reactions were monitored by thin-layer chromatography and UV-vis spectroscopy. The 4-(dodecyloxy)benzaldehyde (2a) [40, 41] and 5-(4-pyridyl)-dipyrromethane [42] were synthesized according to the respective reported literature methods.

3,4,5-tris(dodecyloxy)benzaldehyde (2b). A mixture of 3,4,5-trihydroxybenzaldehyde $(1.54 \mathrm{~g}, 10 \mathrm{mmol})$, 1-bromododecane (14.95 g, $60 \mathrm{mmol}), \mathrm{K}_{2} \mathrm{CO}_{3}(4.16 \mathrm{~g}$, $30 \mathrm{mmol})$, and $\mathrm{KI}(100 \mathrm{mg})$ in DMF $(60 \mathrm{~mL})$ was stirred at $70{ }^{\circ} \mathrm{C}$ for $16 \mathrm{~h}$. The reaction mixture was cooled to room temperature and mixed with water. The resulting aqueous layer was extracted three times with chloroform. The organic layers were combined, then washed with brine and dried over $\mathrm{Na}_{2} \mathrm{SO}_{4}$. The solvent was removed under reduced pressure and the resulting crude product was purified by column chromatography (silica gel, eluent $\mathrm{CHCl}_{3}$ /heptane 2/3) followed by recrystallization from $\mathrm{CHCl}_{3} / \mathrm{MeOH}$. The product was obtained as a white solid (6.26 g, yield: 95\%). ${ }^{1} \mathrm{H}$ NMR (300 MHz, $\mathrm{CDCl}_{3}$ ): $\delta$, ppm 9.85 (s, 1H, -CHO), $7.10(\mathrm{~s}, 2 \mathrm{H}$, phenyl-H), 4.06 $\left(\mathrm{m}, 6 \mathrm{H}, \mathrm{OCH}_{2^{-}}\right), 1.87\left(\mathrm{~m}, 6 \mathrm{H}, \mathrm{OCH}_{2}-\mathrm{CH}_{2}-\right), 1.47(\mathrm{~m}, 6 \mathrm{H}$, $\left.\left.\mathrm{OCH}_{2}-\mathrm{CH}_{2}-\mathrm{CH}_{2}-\right), 1.29\left(\mathrm{~m}, 48 \mathrm{H},-\left(\mathrm{CH}_{2}\right)_{9}\right)^{-}\right), 0.90$ (t, $9 \mathrm{H}$, $J=6 \mathrm{~Hz})$.

5-(4-pyridyl)-10,15,20-tris(4-dodecyloxyphenyl)porphyrin (3a) and 5,15-di(4-pyridyl)-10,20-bis(4dodecyloxyphenyl)porphyrin (4a). 4-(dodecyloxy)benzaldehyde (2.35 mmol, $1.548 \mathrm{~g})$ and 4-pyridinedipyrromethane $(2.35 \mathrm{mmol}, 0.524 \mathrm{~g})$ were dissolved in propionic acid $(30 \mathrm{~mL})$ in a $100 \mathrm{~mL}$ round-bottom flask equipped with a magnetic stirring bar and a water-cooled reflux condenser. The mixture was then allowed to reflux for $2.5 \mathrm{~h}$ under air in the dark. After cooling, the reaction mixture was slowly poured into a $1 / 1(\mathrm{v} / \mathrm{v})$ mixture of methanol $(60 \mathrm{~mL})$ and cold $\left(<5{ }^{\circ} \mathrm{C}\right)$ aqueous $\mathrm{NH}_{4} \mathrm{OH}(60$ $\mathrm{mL}$ ) was added. Filtration and washing with cold water gave a black solid. The resulting residue was purified by silica-gel column chromatography repeatedly with $100 \%$ $\mathrm{CH}_{2} \mathrm{Cl}_{2}, 2 \% \mathrm{MeOH} / \mathrm{CH}_{2} \mathrm{Cl}_{2}$ to afford 5,10,15,20-tetra[4-(dodecyloxy)phenyl]porphyrin as the first band (very small amount), the porphyrin 3a (130 mg, yield: $14 \%$ based on dipyrromethane) as the second band and the porphyrin $4 \mathbf{a}(175 \mathrm{mg}$, yield: $15 \%)$ as the third band.

5-(4-pyridyl)-10,15,20-tris(4-dodecyloxyphenyl)porphyrin (3a). ${ }^{1} \mathrm{H} \mathrm{NMR}\left(\mathrm{CDCl}_{3}\right): \delta$, ppm $9.05(\mathrm{~d}$, $2 \mathrm{H}, J=6 \mathrm{~Hz}$, pyridyl-H), $8.93(\mathrm{~m}, 6 \mathrm{H}$, pyridyl-H and $\beta$-pyrrole-H), 8.80 (d, 2H, $J=6 \mathrm{~Hz}, \beta$-pyrrole-H), 8.19 (d, $2 \mathrm{H}, J=6 \mathrm{~Hz}, \beta$-pyrrole-H), $8.13(\mathrm{~d}, 6 \mathrm{H}, J=9 \mathrm{~Hz}$, phenyl-H), $7.30(\mathrm{~d}, 6 \mathrm{H}, J=9 \mathrm{~Hz}$, phenyl-H), $4.27(\mathrm{t}, 6 \mathrm{H}$, $\left.\mathrm{J}=6 \mathrm{~Hz}, \mathrm{O}-\mathrm{CH}_{2}-\right), 1.99\left(\mathrm{~m}, 6 \mathrm{H}, \mathrm{O}-\mathrm{CH}_{2}-\mathrm{CH}_{2}-\right), 1.64(\mathrm{~m}$, $\left.6 \mathrm{H},-\mathrm{O}-\mathrm{CH}_{2}-\mathrm{CH}_{2}-\mathrm{CH}_{2}-\right), 1.43\left(\mathrm{~m}, 48 \mathrm{H},-\left(\mathrm{CH}_{2}\right)_{8}-\right), 0.92$ (m, 9H, $-\mathrm{CH}_{3}$ ), -2.75 (s, 2H, NH). HR-MS (MALDITOF): $m / z 1168.8084[\mathrm{M}+\mathrm{H}]^{+} 1168.7977$ calcd. for $\mathrm{C}_{79} \mathrm{H}_{102} \mathrm{~N}_{5} \mathrm{O}_{3}$. UV-vis $\left(\mathrm{CH}_{2} \mathrm{Cl}_{2}\right): \lambda_{\max }, \mathrm{nm}\left(\varepsilon \times 10^{-3} \mathrm{M}^{-1}\right.$. $\mathrm{cm}^{-1}$ ) 421 (1769), 517.0 (71), 555.0 (46), 592.1 (26), 650.0 (27).

5,15-di(4-pyridyl)-10,20-bis(4-dodecyloxyphenyl)porphyrin (4a). ${ }^{1} \mathrm{H} \mathrm{NMR}\left(\mathrm{CDCl}_{3}\right)$ : $\delta$, ppm $9.06(\mathrm{~d}, 4 \mathrm{H}$, 


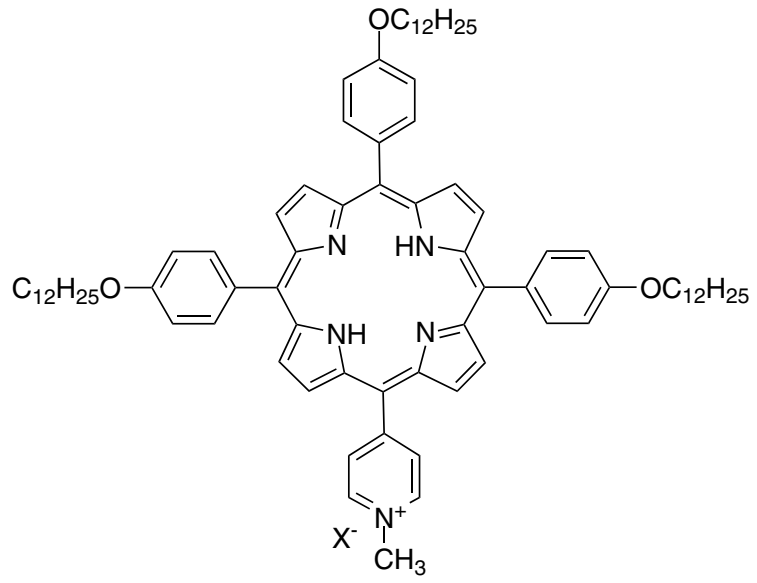

5a: $\mathrm{X}^{-}=\mathrm{I}^{-}$

6a: $\mathrm{X}^{-}=\mathrm{B}\left(\mathrm{C}_{6} \mathrm{~F}_{5}\right)_{4}^{-}$

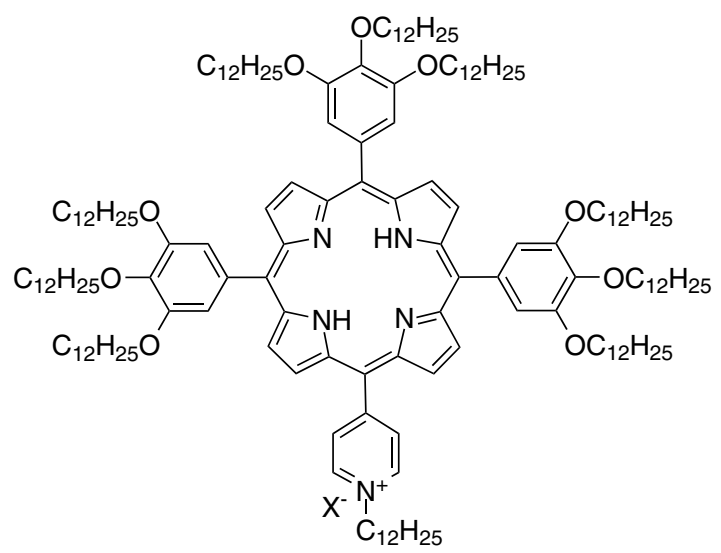

7: $\mathrm{X}^{-}=\mathrm{Br}^{-}$

8: $\mathrm{X}^{-}=\mathrm{B}\left(\mathrm{C}_{6} \mathrm{~F}_{5}\right)_{4}^{-}$

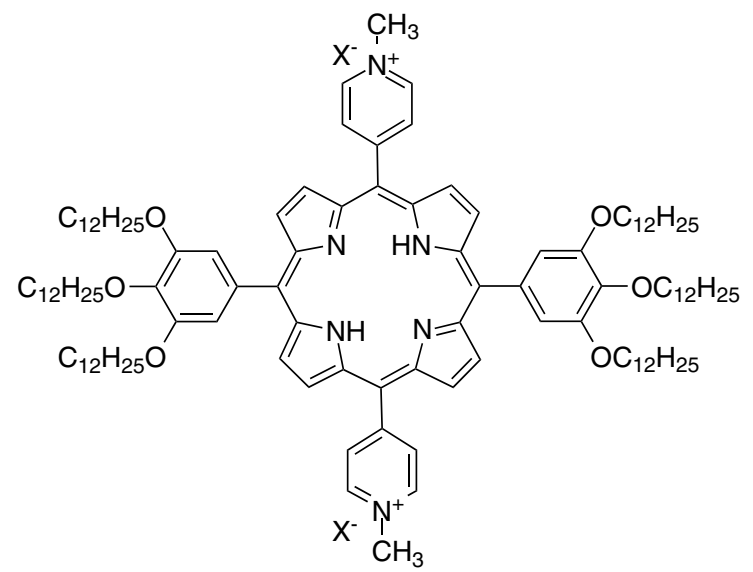

9b: $X^{-}=1^{-}$

10: $\mathrm{X}^{-}=\mathrm{B}\left(\mathrm{C}_{6} \mathrm{~F}_{5}\right)_{4}^{-}$

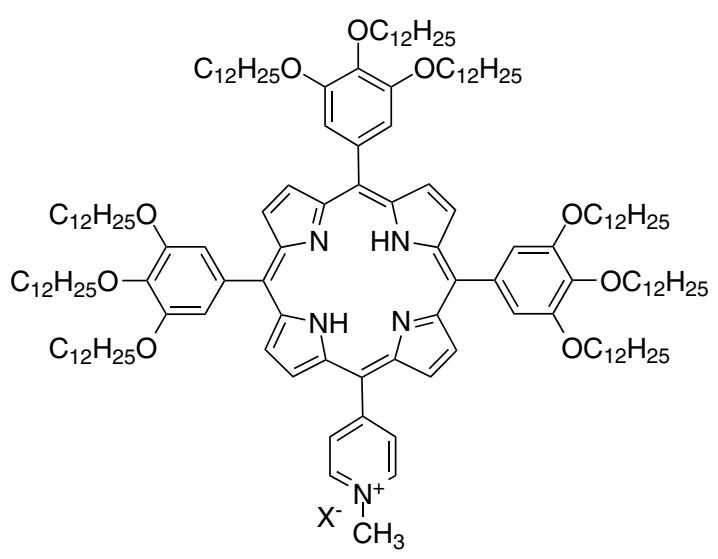

$5 b: X^{-}=I^{-}$

6b: $\mathrm{X}^{-}=\mathrm{B}\left(\mathrm{C}_{6} \mathrm{~F}_{5}\right)_{4}^{-}$

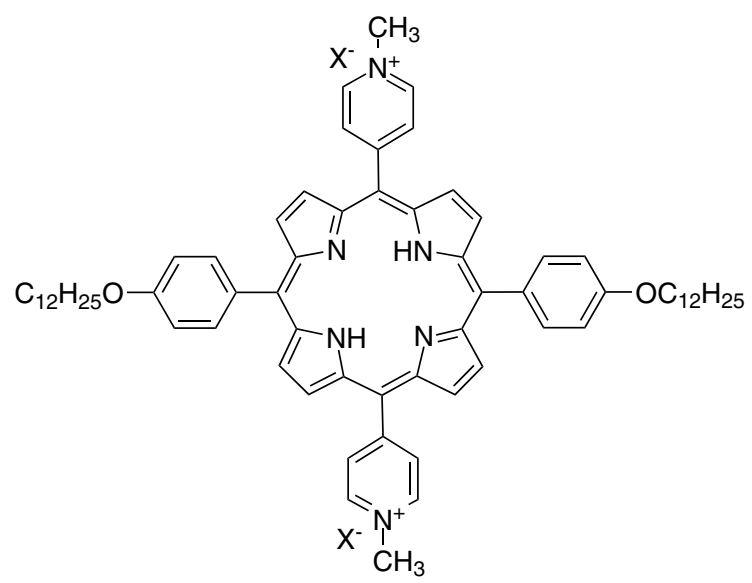

9a: $\mathrm{X}^{-}=\mathrm{I}^{-}$

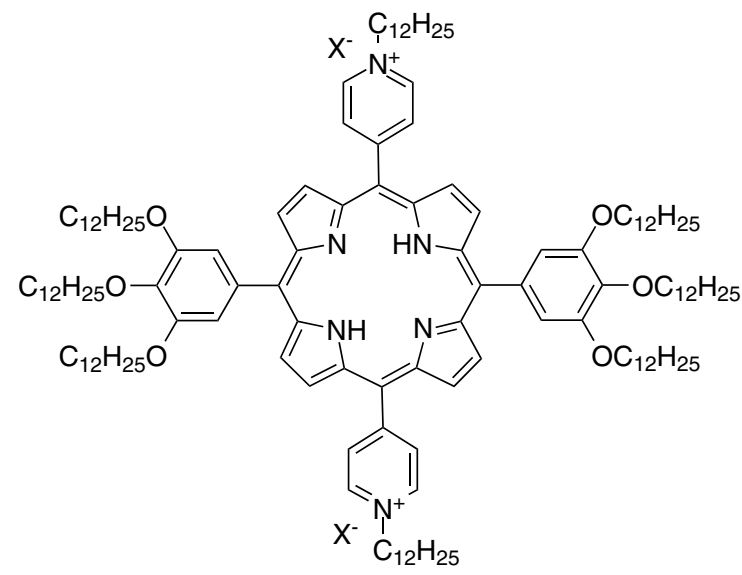

11: $\mathrm{X}^{-}=\mathrm{Br}^{-}$

12: $\mathrm{X}^{-}=\mathrm{B}\left(\mathrm{C}_{6} \mathrm{~F}_{5}\right)_{4}^{-}$

Chart 1. Structures of compounds 5-12 
$J=6 \mathrm{~Hz}$, pyridyl-H), $8.97(\mathrm{~d}, 4 \mathrm{H}, J=6 \mathrm{~Hz}$, pyridyl-H), $8.83(\mathrm{~d}, 4 \mathrm{H}, J=3 \mathrm{~Hz}, \beta$-pyrrole- $\mathrm{H}), 8.18(\mathrm{~d}, 4 \mathrm{H}, J=6 \mathrm{~Hz}$, $\beta$-pyrrole -H), 8.12 (d, $4 \mathrm{H}, J=9 \mathrm{~Hz}$, phenyl-H), 7.31 (d, $4 \mathrm{H}, J=6 \mathrm{~Hz}$, phenyl-H), $4.27\left(\mathrm{t}, 4 \mathrm{H}, J=6.0 \mathrm{~Hz}, \mathrm{O}-\mathrm{CH}_{2^{-}}\right.$), $2.04\left(\mathrm{~m}, 4 \mathrm{H}, \mathrm{O}-\mathrm{CH}_{2}-\mathrm{CH}_{2}-\right), 1.66\left(\mathrm{~m}, 4 \mathrm{H},-\mathrm{O}-\mathrm{CH}_{2}-\mathrm{CH}_{2}-\right.$ $\left.\left.\mathrm{CH}_{2^{-}}\right), 1.44\left(\mathrm{~m}, 32 \mathrm{H},-\left(\mathrm{CH}_{2}\right)_{8}\right)^{-}\right), 0.93(\mathrm{t}, 6 \mathrm{H}, \mathrm{J}=6 \mathrm{~Hz}$, $\left.-\mathrm{CH}_{3}\right),-2.79$ (s, 2H). MS (MALDI-TOF): $\mathrm{m} / 2.985 .61[\mathrm{M}+$ $\mathrm{H}]^{+}, 985.61$ calcd. for $\mathrm{C}_{66} \mathrm{H}_{77} \mathrm{~N}_{6} \mathrm{O}_{2}$. UV-vis $\left(2 \% \mathrm{CH}_{3} \mathrm{OH}\right.$ in $\left.\mathrm{CH}_{2} \mathrm{Cl}_{2}\right): \lambda_{\text {max }}, \mathrm{nm}\left(\varepsilon \times 10^{-3} \mathrm{M}^{-1} \cdot \mathrm{cm}^{-1}\right) 420.0$ (1622), 515.0 (72), 552.0 (37), 591.0 (23), 650.0 (18).

5-(4-pyridyl)-10,15,20-[3,4,5-tris(dodecyloxy)phenyl]porphyrin (3b) and 5,15-dipyridyl-10,20-bis[3,4,5tris(dodecyloxy)phenyl]porphyrin (4b). The reaction was carried out using the same procedure as for $\mathbf{3 a}$ and 4a starting from 3,4,5-tris(dodecyloxy)benzaldehyde $(2.35 \mathrm{mmol}, 1.548 \mathrm{~g})$ and 4-pyridinedipyrromethane $(2.35 \mathrm{mmol}, 0.524 \mathrm{~g})$. The resulting residue was purified by silica-gel column chromatography three times with $100 \% \mathrm{CH}_{2} \mathrm{Cl}_{2}, 25 \% \mathrm{CHCl}_{3}-75 \% \mathrm{CH}_{2} \mathrm{Cl}_{2}$ to afford the purple porphyrin $\mathbf{3 b}$ as the second red band and the purple porphyrin $\mathbf{4 b}$ as the third red band which were both recrystallized from chloroform/methanol to give the pure porphyrin $\mathbf{3 b}$ (143 $\mathrm{mg}$, yield: $8 \%$, according to the dipyrromethane) and the pure porphyrin $\mathbf{4 b}(182 \mathrm{mg}$, yield: $9 \%)$ respectively.

5-(4-pyridyl)-10,15,20-tris[3,4,5-tris(dodecyloxy) phenyl]porphyrin (3b). ${ }^{1} \mathrm{H}$ NMR $\left(\mathrm{CDCl}_{3}\right): \delta$, ppm 9.00 (m, $8 \mathrm{H}$, pyridyl- $\mathrm{H}, \beta$-pyrrole- $\mathrm{H}), 8.79(\mathrm{~d}, 2 \mathrm{H}, J=6 \mathrm{~Hz}$, $\beta$-pyrrole-H), 8.16 (d, 2H, $J=6 \mathrm{~Hz}, \beta$-pyrrole-H), 7.45 (s, 6H, phenyl-H), $4.33\left(\mathrm{t}, 6 \mathrm{H}, \mathrm{J}_{\mathrm{HH}}=6 \mathrm{~Hz}, \mathrm{O}-\mathrm{CH}_{2}-\right), 4.12$ $\left(\mathrm{t}, 12 \mathrm{H},{ }^{3} J_{\mathrm{HH}}=6.6 \mathrm{~Hz},{ }^{3} J_{\mathrm{HH}}=6.3 \mathrm{~Hz}, \mathrm{O}-\mathrm{CH}_{2^{-}}\right), 2.07(\mathrm{~m}$, $\left.6 \mathrm{H}, \mathrm{O}-\mathrm{CH}_{2}-\mathrm{CH}_{2}-\right), 1.88\left(\mathrm{~m}, 12 \mathrm{H}, \mathrm{O}-\mathrm{CH}_{2}-\mathrm{CH}_{2}-\right), 1.72-$ $1.25\left(\mathrm{~m}, 162 \mathrm{H},-\left(\mathrm{CH}_{2}\right)_{9}-\right), 0.90\left(\mathrm{~m}, 27 \mathrm{H},-\mathrm{CH}_{3}\right),-2.80(\mathrm{~s}$, 2H, NH). MS (MALDI-TOF): $m / z 2273.75[\mathrm{M}+\mathrm{H}]^{+}$, 2273.89 calcd. for $\mathrm{C}_{151} \mathrm{H}_{246} \mathrm{~N}_{5} \mathrm{O}_{9}$. UV-vis $\left(\mathrm{CH}_{2} \mathrm{Cl}_{2}\right)$ : $\lambda_{\max }$, $\mathrm{nm}\left(\varepsilon \times 10^{-3} \mathrm{M}^{-1} . \mathrm{cm}^{-1}\right) 421.0$ (350), 516.0 (18), 552.0 (8.7), 591.0 (4.8), 649.0 (4.2).

5,15-di-(4-pyridyl)-10,20-bis[3,4,5-tris(dodecyloxy)phenyl]porphyrin (4b). ${ }^{1} \mathrm{H}$ NMR $\left(\mathrm{CDCl}_{3}\right): \delta$, ppm $9.06(\mathrm{~m}, 8 \mathrm{H}$, pyridyl-H), $8.83(\mathrm{~d}, 4 \mathrm{H}, J=3 \mathrm{~Hz}$, $\beta$-pyrrole-H), 8.19 (d, 4H, $J=6 \mathrm{~Hz}, \beta$-pyrrole-H), 7.45 (s, 6H, phenyl-H), 4.33 (t, 4H, $\left.J=6 \mathrm{~Hz}, \mathrm{O}-\mathrm{CH}_{2^{-}}\right), 4.12(\mathrm{t}$, $\left.8 \mathrm{H}, \mathrm{J}=6 \mathrm{~Hz}, \mathrm{O}-\mathrm{CH}_{2}-\right), 2.01\left(\mathrm{~m}, 4 \mathrm{H}, \mathrm{O}-\mathrm{CH}_{2}-\mathrm{CH}_{2}-\right), 1.90$ (m, 8H, O- $\left.\left.\mathrm{CH}_{2}-\mathrm{CH}_{2}\right)^{-}\right), 1.25-1.67$ (m, $108 \mathrm{H},-\left(\mathrm{CH}_{2}\right)_{9} 9^{-}$), 0.95-0.85 (m, 18H), -2.82 (s, 2H). HR-MS (MALDITOF): $m / z, 1722.3385[\mathrm{M}+\mathrm{H}]^{+}, 1722.3411$ calcd. for $\mathrm{C}_{114} \mathrm{H}_{173} \mathrm{~N}_{6} \mathrm{O}_{6}$. UV-vis $\left(\mathrm{CH}_{2} \mathrm{Cl}_{2}\right): \lambda_{\text {max }}, \mathrm{nm}\left(\varepsilon \times 10^{-3} \mathrm{M}^{-1}\right.$. $\mathrm{cm}^{-1}$ ) 421.0 (448.9), 516.0 (22.7), 550.0 (8.8), 591.0 (7.0), 646.0 (4.6).

5-( $N$-methylpyridinium-4-yl)-10,15,20-tris(4-dodecyloxyphenyl)porphyrin iodide (5a). $50 \mathrm{mg}$ of $\mathbf{3 a}$ $(0.042 \mathrm{mmol})$ was dissolved in $40 \mathrm{~mL}$ of chloroform and $5 \mathrm{~mL}$ of DMF, $2 \mathrm{~mL}$ of methyl iodide was added to the solution of $\mathbf{3 a}$, and the mixture was then stirred under argon at room temperature overnight and monitored by TLC. After the end of the reaction, the solution was concentrated to about $3 \mathrm{~mL}$ and then $50 \mathrm{~mL}$ of ether was poured into the mixture. The precipitate was then filtered and washed with cold chloroform. The precipitate was further purified by column chromatography on silica gel with $10 \% \mathrm{CH}_{3} \mathrm{OH}$ in $\mathrm{CH}_{2} \mathrm{Cl}_{2}$. Yield: $55 \mathrm{mg}, 98 \%$. mp 181-182 ${ }^{\circ} \mathrm{C} .{ }^{1} \mathrm{H} \mathrm{NMR}\left(\mathrm{CDCl}_{3}\right): \delta$, ppm $9.42(\mathrm{~d}, 2 \mathrm{H}, J=6$ $\mathrm{Hz}$, pyridyl-H), 8.89 (d, 2H, $J=6 \mathrm{~Hz}$, pyridyl-H), 8.85 (d, $2 \mathrm{H}, J=6 \mathrm{~Hz}, \beta$-pyrrole- $\mathrm{H}), 8.78$ (m, 4H, $\beta$-pyrrole- $\mathrm{H}$ ), $8.63(\mathrm{~d}, 2 \mathrm{H}, J=6 \mathrm{~Hz}, \beta$-pyrrole-H), 8.06 (d, 2H, $J=9 \mathrm{~Hz}$, phenyl-H), 7.88 (d, 4H, $J=9 \mathrm{~Hz}$, phenyl-H), $7.29(\mathrm{~d}, 2 \mathrm{H}$, $J=9 \mathrm{~Hz}$, phenyl-H), $7.11(\mathrm{~d}, 4 \mathrm{H}, J=9 \mathrm{~Hz}$, phenyl-H), $4.93\left(\mathrm{~s}, 3 \mathrm{H}, \mathrm{N}-\mathrm{CH}_{3}\right), 4.26\left(\mathrm{t}, 2 \mathrm{H},{ }^{3} \mathrm{~J}_{\mathrm{HH}}=6.6 \mathrm{~Hz},{ }^{3} J_{\mathrm{HH}}=6.6\right.$ $\left.\mathrm{Hz}, \mathrm{O}-\mathrm{CH}_{2}-\right), 4.11\left(\mathrm{t}, 4 \mathrm{H},{ }^{3} J_{\mathrm{HH}}=6.6 \mathrm{~Hz},{ }^{3} J_{\mathrm{HH}}=6.6 \mathrm{~Hz}\right.$, O- $\left.\mathrm{CH}_{2}{ }^{-}\right), 2.01-1.91\left(\mathrm{~m}, 6 \mathrm{H}, \mathrm{O}-\mathrm{CH}_{2}-\mathrm{CH}_{2}\right), 1.68-1.33$ (m, 54H, $\left.\left.-\left(\mathrm{CH}_{2}\right)_{9}\right)^{-}\right), 0.94-0.89\left(\mathrm{~m}, 9 \mathrm{H},-\mathrm{CH}_{3}\right),-2.74$ (s, 2H). MS (MALDI-TOF): $m / z, 1182.72$ [M - I] ${ }^{+}, 1182.81$ calcd. for $\mathrm{C}_{80} \mathrm{H}_{104} \mathrm{~N}_{5} \mathrm{O}_{3}$. UV-vis $\left(\mathrm{CH}_{2} \mathrm{Cl}_{2}\right): \lambda_{\max }, \mathrm{nm}(\varepsilon \times$ $\left.10^{-3} \mathrm{M}^{-1} . \mathrm{cm}^{-1}\right) 422.0$ (143), 522.0 (11), 584.0 (13), 663.0 (0.8). Anal. calcd. for $\mathrm{C}_{80} \mathrm{H}_{104} \mathrm{IN}_{5} \mathrm{O}_{3}: \mathrm{C} 73.31 ; \mathrm{H} 8.00 ; \mathrm{N}$ 5.34. Found: C 72.77; H 7.96; N 5.58.

5-( $N$-methylpyridinium-4-yl)-10,15,20-tris [3,4,5tris(dodecyloxy)phenyl]porphyrin iodide $(5 \mathrm{~b})$. To a solution of $\mathbf{3 b}(0.022 \mathrm{mmol}, 50 \mathrm{mg})$ dissolved in $40 \mathrm{~mL}$ chloroform and $5 \mathrm{~mL}$ of DMF was added $1.5 \mathrm{~mL}$ of methyl iodide slowly, and the mixture was then stirred under an argon atmosphere at room temperature overnight. The solvent was removed under reduced pressure. The solid residue was purified by column chromatography on a silica gel with $10 \% \mathrm{CH}_{3} \mathrm{OH}$ in $\mathrm{CHCl}_{3}$. The product was recrystallized from $\mathrm{CHCl}_{3} /$ methanol mixture layered by hexane. Yield: $52 \mathrm{mg}, 96 \%$. Liquid. ${ }^{1} \mathrm{H}$ NMR $\left(\mathrm{CDCl}_{3}\right): \delta$, ppm $9.68(\mathrm{~d}, 2 \mathrm{H}, J=6.0 \mathrm{~Hz}$, pyridyl-H), 9.13 (d, 2H, $J=6 \mathrm{~Hz}$, pyridyl-H), 8.99 (m, 4H, $\beta$-pyrrole-H), 8.93 (t, 2H, $J=3 \mathrm{~Hz}, \beta$-pyrrole-H), 8.84 (d, 2H, $J=6$ $\mathrm{Hz}, \beta$-pyrrole-H), 7.43 (s, 6H, phenyl-H), 5.10 (s, 3H, $\left.\mathrm{N}-\mathrm{CH}_{3}\right), 4.33\left(\mathrm{t}, 6 \mathrm{H}, \mathrm{J}=6 \mathrm{~Hz}, \mathrm{O}-\mathrm{CH}_{2}\right), 4.12(\mathrm{t}, 12 \mathrm{H}$, $\left.J=6 \mathrm{~Hz}, \mathrm{O}-\mathrm{CH}_{2}-\right), 2.02-1.85\left(\mathrm{~m}, 18 \mathrm{H}, \mathrm{O}-\mathrm{CH}_{2}-\mathrm{CH}_{2}-\right)$, $1.70\left(\mathrm{~m}, 6 \mathrm{H}, \mathrm{O}-\mathrm{CH}_{2}-\mathrm{CH}_{2}-\mathrm{CH}_{2}-\right), 1.49\left(\mathrm{~m}, 12 \mathrm{H}, \mathrm{O}-\mathrm{CH}_{2}-\right.$ $\left.\mathrm{CH}_{2}-\mathrm{CH}_{2}-\right), 1.32-1.23\left(\mathrm{~m}, 144 \mathrm{H},-\left(\mathrm{CH}_{2}\right)_{8^{-}}\right), 0.92-0.83$ $\left(\mathrm{m}, 27 \mathrm{H},-\mathrm{CH}_{3}\right),-2.71(\mathrm{~s}, 2 \mathrm{H}, \mathrm{NH})$. HR-MS (MALDITOF): $m / z 2287.8661[\mathrm{M}-\mathrm{I}]^{+}, 2287.9096$ calcd. for $\mathrm{C}_{152} \mathrm{H}_{248} \mathrm{~N}_{5} \mathrm{O}_{9}$. UV-vis $\left(\mathrm{CH}_{2} \mathrm{Cl}_{2}\right): \lambda_{\max }, \mathrm{nm}\left(\varepsilon \times 10^{-3} \mathrm{M}^{-1}\right.$. $\left.\mathrm{cm}^{-1}\right) 428.0$ (122), 522.0 (12), 589.0 (1.1), 659.0 (0.6). Anal. calcd. for $\mathrm{C}_{152} \mathrm{H}_{248} \mathrm{IN}_{5} \mathrm{O}_{9}$ : C 75.55; H 10.34; N 2.90 . Found: C 75.90; H 10.30; N 2.85.

5-( $N$-methylpyridinium-4-yl)-10,15,20-tris(4-dodecyloxyphenyl)porphyrin tetrakis(pentafluorophenyl)borate (6a). To a $3 \mathrm{mLCH}_{2} \mathrm{Cl}_{2}$ solution of $\mathrm{NaB}\left(\mathrm{C}_{6} \mathrm{~F}_{5}\right)_{4} \cdot \mathrm{Et}_{2} \mathrm{O}$ (46.4 mg, $0.067 \mathrm{mmol}$ ) was added a $5 \mathrm{~mL} \mathrm{CH}_{2} \mathrm{Cl}_{2}$ solution of $5 \mathbf{a}(80 \mathrm{mg}, 0.067 \mathrm{mmol})$. The reaction mixture was stirred for $48 \mathrm{~h}$ under argon before filtration to remove NaI. The filtrate was concentrated under reduced pressure to give a crude product, which was washed with heptane and sonicated for $1 \mathrm{~h}$. The product was purified by recrystallization $\left(\mathrm{CHCl}_{3} /\right.$ hexane) . Any remaining volatile components were removed under reduced pressure to afford a purple-black solid. Yield: $101 \mathrm{mg}$, 80\%. mp $128-129^{\circ} \mathrm{C} .{ }^{1} \mathrm{H}$ NMR $\left(\mathrm{CDCl}_{3}\right): \delta$, ppm 8.98 
(d, 2H, $J=6 \mathrm{~Hz}$, pyridyl-H), 8.93-8.89 (m, 4H, pyridyl-H, $\beta$-pyrrole-H), 8.61 (d, 2H, $J=9 \mathrm{~Hz}, \beta$-pyrrole-H), 8.51 ( $\mathrm{m}, 4 \mathrm{H}, \beta$-pyrrole- $\mathrm{H}), 8.08$ ( $\mathrm{m}, 6 \mathrm{H}$, phenyl-H), $7.31\left(\mathrm{~m}, 6 \mathrm{H}\right.$, phenyl-H), $4.44\left(\mathrm{~s}, 3 \mathrm{H}, \mathrm{N}-\mathrm{CH}_{3}\right), 4.25(\mathrm{~m}$, $\left.6 \mathrm{H}, \mathrm{O}-\mathrm{CH}_{2}-\right), 1.99\left(\mathrm{~m}, 6 \mathrm{H}, \mathrm{O}-\mathrm{CH}_{2}-\mathrm{CH}_{2}-\right), 1.64-1.28$ (m, 54H, $\left.-\left(\mathrm{CH}_{2}\right)_{9^{-}}\right), 0.92\left(\mathrm{~m}, 9 \mathrm{H},-\mathrm{CH}_{3}\right),-2.51(\mathrm{~s}, 2 \mathrm{H}$, $\mathrm{NH}) .{ }^{19} \mathrm{~F} \mathrm{NMR}\left(\mathrm{CDCl}_{3}\right): \delta$, ppm -132.6 (d, 2F), -162.2 (t, 1F), -166.3 (t, 2F). MS (MALDI-TOF): $\mathrm{m} / z$, 1182.77 $\left[\mathrm{M}-\mathrm{B}\left(\mathrm{C}_{6} \mathrm{~F}_{5}\right)_{4}\right]^{+}, 1182.81$ calcd. for $\mathrm{C}_{80} \mathrm{H}_{104} \mathrm{~N}_{5} \mathrm{O}_{3}$. UV-vis $\left(\mathrm{CH}_{2} \mathrm{Cl}_{2}\right): \lambda_{\max }, \mathrm{nm}\left(\varepsilon \times 10^{-3} \mathrm{M}^{-1} . \mathrm{cm}^{-1}\right) 419.0(131), 590.0$ (1.6), 661.0 (1.1). Anal. calcd. for $\mathrm{C}_{104} \mathrm{H}_{104} \mathrm{BF}_{20} \mathrm{~N}_{5} \mathrm{O}_{3}$ : C 67.06; H 5.63; N 3.76. Found: C 67.07; H 6.03; N 3.83.

5-( $N$-methylpyridinium-4-yl)-10,15,20-tris[3,4,5tris(dodecyloxy)phenyl]porphyrin tetrakis(pentafluorophenyl)borate (6b). To a flask containing NaB$\left(\mathrm{C}_{6} \mathrm{~F}_{5}\right)_{4} \mathrm{Et}_{2} \mathrm{O}(25 \mathrm{mg}, 0.035 \mathrm{mmol})$ was added a solution of $\mathbf{5 b}(60 \mathrm{mg}, 0.025 \mathrm{mmol})$ in $\mathrm{CH}_{2} \mathrm{Cl}_{2}(5 \mathrm{~mL})$. The reaction mixture was stirred for $48 \mathrm{~h}$ under argon before filtration to remove NaI. The filtrate was diluted with $\mathrm{CH}_{2} \mathrm{Cl}_{2}$ and washed with brine, distilled water and dry over $\mathrm{MgSO}_{4}$. The organic solution was concentrated under reduced pressure to give a crude product, which was purified by recrystallization $\left(\mathrm{CHCl}_{3} /\right.$ methanol) to afford the oil porphyrin $(51 \mathrm{mg}, 69 \%) .{ }^{1} \mathrm{H}$ NMR $\left(\mathrm{CDCl}_{3}\right)$ : $\delta$, ppm 9.09 (d, 2H, $J=6 \mathrm{~Hz}$, pyridyl-H), 8.99 (m, 4H, $\beta$-pyrrole-H), 8.85 (s, 4H, $\beta$-pyrrole- $\mathrm{H}), 8.59$ (d, 2H, $J=6 \mathrm{~Hz}$, pyridyl-H), 7.42 (s, 6H, phenyl-H), 4.65 (s, $3 \mathrm{H}$, $\left.\mathrm{N}-\mathrm{CH}_{3}\right), 4.32\left(\mathrm{t}, 6 \mathrm{H}, \mathrm{J}=6 \mathrm{~Hz}, \mathrm{O}-\mathrm{CH}_{2}-\right), 4.10(\mathrm{t}, 12 \mathrm{H}$, $\left.\mathrm{J}=6 \mathrm{~Hz}, \mathrm{O}-\mathrm{CH}_{2}-\right), 1.99\left(\mathrm{~m}, 6 \mathrm{H}, \mathrm{O}-\mathrm{CH}_{2}-\mathrm{CH}_{2}-\right), 1.87(\mathrm{~m}$, $\left.12 \mathrm{H}, \mathrm{O}-\mathrm{CH}_{2}-\mathrm{CH}_{2}-\right), 1.54-1.22\left(\mathrm{~m}, 162 \mathrm{H},-\left(\mathrm{CH}_{2}\right)_{9}{ }^{-}\right)$, 0.91-0.83 (m, 27H, $\left.-\mathrm{CH}_{3}\right),-2.59$ (s, 2H, NH). ${ }^{19} \mathrm{~F}$ NMR $\left(\mathrm{CDCl}_{3}\right): \delta$, ppm -132.6 (d, 2F), -162.2 (t, 1F), -166.3 (t, 2F). HR-MS (MALDI-TOF): $\mathrm{m} / z 2287.9025$ [M $\left.\mathrm{B}\left(\mathrm{C}_{6} \mathrm{~F}_{5}\right)_{4}\right]^{+}, 2287.9097$ calcd. for $\mathrm{C}_{152} \mathrm{H}_{248} \mathrm{~N}_{5} \mathrm{O}_{9}$. UV-vis $\left(\mathrm{CH}_{2} \mathrm{Cl}_{2}\right): \lambda_{\max }, \mathrm{nm}\left(\varepsilon \times 10^{-3} \mathrm{M}^{-1} \cdot \mathrm{cm}^{-1}\right) 421.0(113), 589.0$ (14), 633.0 (0.8). Anal. calcd. for $\mathrm{C}_{176} \mathrm{H}_{248} \mathrm{BF}_{20} \mathrm{~N}_{5} \mathrm{O}_{9}$ : C 71.21 ; H 8.42; N 2.36. Found: C 71.19; H 8.53; N 2.41.

5-(4- $\mathrm{N}$-dodecanoylpyridinium-4-yl)-10,15,20-tris[3,4,5-tris(dodecyloxy)phenyl]porphyrin bromide (7). Porphyrin 3b (200 mg, $0.105 \mathrm{mmol}$ ) was added to $1.5 \mathrm{~g}$ (6 mmol) of 1-bromododecane in $100 \mathrm{~mL}$ of dry DMF and the mixture was then heated to reflux for $6 \mathrm{~h}$ under Ar. After evaporation of the solvent, the residual solid was washed with ether and filtered out. Purification of the solid by chromatography on silica gel with $10 \%$ $\mathrm{CH}_{3} \mathrm{OH}-\mathrm{CH}_{2} \mathrm{Cl}_{2}$ gave $217 \mathrm{mg}$ product. Yield: $96 \%$. mp 145-146 ${ }^{\circ} \mathrm{C} .{ }^{1} \mathrm{H}$ NMR $\left(\mathrm{CDCl}_{3}\right): \delta$, ppm $9.78(\mathrm{~d}, 2 \mathrm{H}, J=$ $6 \mathrm{~Hz}$, pyridyl-H), 9.12 (d, 2H, $J=6 \mathrm{~Hz}$, pyridyl-H), 8.99 (s, 4H, $\beta$-pyrrole-H), 8.85 (t, 4H, $J=6 \mathrm{~Hz}, \beta$-pyrrole- $\mathrm{H}$ ), $7.43\left(\mathrm{~s}, 6 \mathrm{H}\right.$, phenyl-H), $5.37\left(\mathrm{t}, 2 \mathrm{H},{ }^{3} \mathrm{~J}_{\mathrm{HH}}=6 \mathrm{~Hz},{ }^{3} J_{\mathrm{HH}}=\right.$ $\left.9 \mathrm{~Hz}, \mathrm{~N}-\mathrm{CH}_{2}-\right), 4.32\left(\mathrm{t}, 6 \mathrm{H}, \mathrm{J}=6 \mathrm{~Hz}, \mathrm{O}-\mathrm{CH}_{2^{-}}\right), 4.12(\mathrm{t}$, $\left.12 \mathrm{H}, \mathrm{J}=6 \mathrm{~Hz}, \mathrm{O}-\mathrm{CH}_{2}-\right), 2.40\left(\mathrm{~m}, 2 \mathrm{H}, \mathrm{N}-\mathrm{CH}_{2}-\mathrm{CH}_{2}-\right), 2.00$ (m, 6H, O- $\mathrm{CH}_{2}-\mathrm{CH}_{2}-$ ), $1.91-1.87$ (m, $12 \mathrm{H}, \mathrm{O}-\mathrm{CH}_{2}-\mathrm{CH}_{2}$ ), 1.67-1.23 (m, $\left.\left.180 \mathrm{H},-\left(\mathrm{CH}_{2}\right)_{9}\right)^{-}\right), 0.93-0.83(\mathrm{~m}, 30 \mathrm{H}$, $\left.-\mathrm{CH}_{3}\right),-2.70(\mathrm{~s}, 2 \mathrm{H}, \mathrm{NH})$. MS (MALDI-TOF): $\mathrm{m} / \mathrm{z}$ $2442.15[\mathrm{M}-\mathrm{Br}]^{+}, 2442.08$ calcd. for $\mathrm{C}_{163} \mathrm{H}_{270} \mathrm{~N}_{5} \mathrm{O}_{9}$. UVvis $\left(\mathrm{CH}_{2} \mathrm{Cl}_{2}\right): \lambda_{\max }, \mathrm{nm}\left(\varepsilon \times 10^{-3} \mathrm{M}^{-1} \cdot \mathrm{cm}^{-1}\right) 427.0$ (131),
524.0 (13), 593.0 (13), 659.0 (0.7). Anal. calcd. for $\mathrm{C}_{163} \mathrm{H}_{270} \mathrm{BrN}_{5} \mathrm{O}_{9} \cdot 3 \mathrm{H}_{2} \mathrm{O}: \mathrm{C}$ 76.77; $\mathrm{H} 10.29 ; \mathrm{N} 2.65$. Found: C 76.90; H 10.26; N 2.80.

5-(4- $N$-dodecanoylpyridinium-4-yl)-10,15,20-tris[3,4,5-tris(dodecyloxy)phenyl]porphyrin tetrakis(pentafluorophenyl)borate (8). To a $3 \mathrm{~mL} \mathrm{CH}_{2} \mathrm{Cl}_{2}$ solution of $\mathrm{NaB}\left(\mathrm{C}_{6} \mathrm{~F}_{5}\right)_{4} \cdot \mathrm{Et}_{2} \mathrm{O}(30.1 \mathrm{mg}, 0.04 \mathrm{mmol})$ was added a $5 \mathrm{~mL}$ of $\mathrm{CH}_{2} \mathrm{Cl}_{2}$ solution of $7(100 \mathrm{mg}, 0.04 \mathrm{mmol})$. The reaction mixture was stirred for $48 \mathrm{~h}$ under argon. The solution was diluted with $\mathrm{CH}_{2} \mathrm{Cl}_{2}$. The organic solution was washed with distilled water, dried over $\mathrm{MgSO}_{4}$, and the solvent was removed under reduced pressure. The product was purified by recrystallization $\left(\mathrm{CHCl}_{3} /\right.$ methanol $)$ to afford an oily black product (104 mg, yield: 84\%). Liquid. ${ }^{1} \mathrm{H}$ NMR $\left(\mathrm{CDCl}_{3}\right): \delta$, ppm 9.09 (d, 2H, $J=6 \mathrm{~Hz}$, pyridyl-H), 8.99 (m, 4H, $\beta$-pyrrole-H), 8.90 (d, 2H, $J=9$ $\mathrm{Hz}, \beta$-pyrrole-H), 8.84 (d, 2H, $J=6.0 \mathrm{~Hz}, \beta$-pyrrole-H), 8.62 (d, $2 \mathrm{H}, J=3 \mathrm{~Hz}$, pyridyl-H), 7.43 (s, $6 \mathrm{H}$, phenyl-H), $4.78\left(\mathrm{t}, 2 \mathrm{H}, J=6 \mathrm{~Hz}, \mathrm{~N}-\mathrm{CH}_{2^{-}}\right), 4.32(\mathrm{t}, 6 \mathrm{H}, J=6.0 \mathrm{~Hz}$, $\left.\mathrm{O}-\mathrm{CH}_{2}-\right), 4.10\left(\mathrm{~m}, 12 \mathrm{H}, \mathrm{O}-\mathrm{CH}_{2}-\right), 2.31\left(\mathrm{~m}, 2 \mathrm{H}, \mathrm{N}-\mathrm{CH}_{2}-\right.$ $\left.\mathrm{CH}_{2}{ }^{-}\right), 1.98$ (m, 6H, O- $\left.\mathrm{CH}_{2}-\mathrm{CH}_{2}{ }^{-}\right), 1.90$ (m, $12 \mathrm{H}, \mathrm{O}_{-} \mathrm{CH}_{2}-$ $\left.\left.\mathrm{CH}_{2^{-}}\right), 1.70-1.23\left(\mathrm{~m}, 180 \mathrm{H},-\left(\mathrm{CH}_{2}\right)_{9}\right)^{-}\right), 0.92-0.84$ (m, $\left.30 \mathrm{H},-\mathrm{CH}_{3}\right),-2.59(\mathrm{~s}, 2 \mathrm{H}, \mathrm{NH}) .{ }^{19} \mathrm{~F} \mathrm{NMR}\left(\mathrm{CDCl}_{3}\right): \delta$, ppm -132.5 (t, 2F), -162.4 (m, 1F), -166.52 (m, 2F). MS (MALDI-TOF): $m / z, 2442.00\left[\mathrm{M}-\mathrm{B}\left(\mathrm{C}_{6} \mathrm{~F}_{5}\right)_{4}\right]^{+}, 2442.08$ calcd. for $\mathrm{C}_{163} \mathrm{H}_{270} \mathrm{~N}_{5} \mathrm{O}_{9}$. UV-vis $\left(\mathrm{CH}_{2} \mathrm{Cl}_{2}\right)$ : $\lambda_{\max }, \mathrm{nm}(\varepsilon \times$ $\left.10^{-3} \mathrm{M}^{-1} . \mathrm{cm}^{-1}\right) 423.0$ (118), 593.0 (15), 664.0 (0.9). Anal. calcd. for $\mathrm{C}_{163} \mathrm{H}_{270} \mathrm{BF}_{20} \mathrm{~N}_{5} \mathrm{O}_{9}$ : C 71.92; H 8.71; N 2.24. Found: C 72.14; H 8.82; N 2.35 .

5,15-di( $N$-methylpyridinium-4-yl)-10,20-bis(4-dodecyloxyphenyl)porphyrin diiodide (9a). To a solution of $4 \mathbf{a}(50 \mathrm{mg}, 0.05 \mathrm{mmol})$ dissolved in $45 \mathrm{~mL}$ of chloroform and $5 \mathrm{~mL}$ of DMF was added $2 \mathrm{~mL}$ of methyl iodide, and the reaction mixture was stirred under argon at room temperature for two days and monitored by TLC. The solution was then concentrated to about $3 \mathrm{~mL}$ and then $50 \mathrm{~mL}$ of ether was poured into the mixture. The precipitate was then filtered and washed with chloroform. After drying of the precipitate under vacuum, it was recrystallized from mixed solvents of chloroform and methanol with layered hexane. Yield: $61 \mathrm{mg}, 96 \%$. mp 235-236 ${ }^{\circ} \mathrm{C} .{ }^{1} \mathrm{H}$ NMR (DMSO-d 6 ): $\delta$, ppm 9.47 (d, 4H, $J=6 \mathrm{~Hz}$, pyridyl-H), $9.01(\mathrm{~d}, 12 \mathrm{H}, J=6 \mathrm{~Hz}$, pyridyl-H and $\beta$-pyrrole-H), 8.12 (d, $4 \mathrm{H}, J=9 \mathrm{~Hz}$, phenyl-H), 7.41 (d, $4 \mathrm{H}, J=9 \mathrm{~Hz}$, phenyl-H), $4.72\left(\mathrm{~s}, 6 \mathrm{H}, \mathrm{N}-\mathrm{CH}_{3}\right), 4.27$ (t, $\left.4 \mathrm{H}, \mathrm{J}=6 \mathrm{~Hz}, \mathrm{OCH}_{2^{-}}\right), 1.91\left(\mathrm{~m}, 4 \mathrm{H}, \mathrm{OCH}_{2}-\mathrm{CH}_{2}-\right), 1.57$ (m, $\left.4 \mathrm{H}, \mathrm{OCH}_{2}-\mathrm{CH}_{2}-\mathrm{CH}_{2}-\right), 1.47-1.21\left(\mathrm{~m}, 32 \mathrm{H},-\left(\mathrm{CH}_{2}\right)_{8^{-}}\right)$, $0.86\left(\mathrm{~m}, 6 \mathrm{H},-\mathrm{CH}_{3}\right),-2.94(\mathrm{~s}, 2 \mathrm{H}, \mathrm{NH})$. MS (MALDITOF): $m / z 1015.62[\mathrm{M}-2 \mathrm{I}+\mathrm{H}]^{+}, 1015.66$ calcd. for $\mathrm{C}_{68} \mathrm{H}_{83} \mathrm{~N}_{6} \mathrm{O}_{2}$. UV-vis $\left(\mathrm{CH}_{2} \mathrm{Cl}_{2}\right): \lambda_{\text {max }}, \mathrm{nm}\left(\varepsilon \times 10^{-3} \mathrm{M}^{-1}\right.$. $\left.\mathrm{cm}^{-1}\right) 438.0$ (115), 526.0 (9.4), 574.0 (11), 666.0 (7.5). Anal. calcd. for $\mathrm{C}_{68} \mathrm{H}_{82} \mathrm{I}_{2} \mathrm{~N}_{6} \mathrm{O}_{2} \cdot 4 \mathrm{H}_{2} \mathrm{O}: \mathrm{C} 60.89 ; \mathrm{H} 6.76 ; \mathrm{N}$ 6.27. Found: C 60.89; H 6.36; N 6.46.

5,15-di( $N$-methylpyridinium-4-yl)-10,20-bis[3,4,5tris(dodecyloxy)phenyl]porphyrin diiodide (9b). To $100 \mathrm{mg}$ of 5,15-dipyridyl-10,20-bis(3,4,5-tris(dodecyloxy)phenyl)porphyrin $(0.058 \mathrm{mmol})$ dissolved in $45 \mathrm{~mL}$ 
of chloroform and $5 \mathrm{~mL}$ of DMF was slowly added $2.0 \mathrm{~mL}$ of methyl iodide, and the mixture was then stirred under an argon atmosphere at room temperature overnight. The solvent was removed under reduced pressure. The solid residue was purified by column chromatography on a silica gel using $10 \% \mathrm{CH}_{3} \mathrm{OH}-\mathrm{CHCl}_{3}$ as eluent. The product was recrystallized from $\mathrm{CHCl}_{3} /$ methanol/hexane. Yield: $96 \%$ (111 mg). mp 181-183 ${ }^{\circ} \mathrm{C} .{ }^{1} \mathrm{H} \mathrm{NMR}\left(\mathrm{CDCl}_{3}\right)$ : $\delta$, ppm $9.66(\mathrm{~d}, 4 \mathrm{H}, J=6 \mathrm{~Hz}$, pyridyl-H), $9.12(\mathrm{~d}, 4 \mathrm{H}, J=$ $3 \mathrm{~Hz}$, pyridyl-H), 9.00 (s, 4H, $\beta$-pyrrole-H), 8.77 (d, 4H, $J=6.0 \mathrm{~Hz}, \beta$-pyrrole-H), 7.38 (s, 4H, phenyl-H), 5.08 $\left(\mathrm{s}, 6 \mathrm{H}, \mathrm{N}-\mathrm{CH}_{3}\right), 4.32$ (t, $\left.4 \mathrm{H}, \mathrm{J}=6 \mathrm{~Hz}, \mathrm{O}-\mathrm{CH}_{2^{-}}\right), 4.11$ (t, $\left.8 \mathrm{H}, \mathrm{J}=6 \mathrm{~Hz}, \mathrm{O}-\mathrm{CH}_{2}-\right), 2.01\left(\mathrm{~m}, 4 \mathrm{H}, \mathrm{O}-\mathrm{CH}_{2}-\mathrm{CH}_{2}-\right), 1.90$ (m, 8H, O- $\left.\mathrm{CH}_{2}-\mathrm{CH}_{2}{ }^{-}\right), 1.72-1.20\left(\mathrm{~m}, 108 \mathrm{H},-\left(\mathrm{CH}_{2}\right)_{9}\right)^{-}$), 0.91-0.80 (m, $\left.18 \mathrm{H},-\mathrm{CH}_{3}\right),-3.15$ (s, 2H, NH). HR-MS (MALDI-TOF): $m / z$ 1752.3856 [M - 2I + H] $]^{+}, 1752.3881$ calcd. for $\mathrm{C}_{116} \mathrm{H}_{179} \mathrm{~N}_{6} \mathrm{O}_{6}$. UV-vis $\left(\mathrm{CH}_{2} \mathrm{Cl}_{2}\right): \lambda_{\max }, \mathrm{nm}(\varepsilon \times$ $\left.10^{-3} \mathrm{M}^{-1} . \mathrm{cm}^{-1}\right) 441.0$ (220), 526.0 (17.9), 575.1 (18.1), 663.0 (11.1). Anal. calcd. for $\mathrm{C}_{116} \mathrm{H}_{178} \mathrm{I}_{2} \mathrm{~N}_{6} \mathrm{O}_{6}: \mathrm{C} 69.44 ; \mathrm{H}$ 8.94; N 4.19. Found: C 69.46; H 8.75; N 4.26.

5,15-di( $N$-methylpyridinium4-yl)-10,20-bis[3,4,5tris(dodecyloxy)phenyl]porphyrin di-[tetrakis(pentafluorophenyl)borate] (10). To a $3 \mathrm{~mL} \mathrm{CH}_{2} \mathrm{Cl}_{2}$ solution of $\mathrm{NaB}\left(\mathrm{C}_{6} \mathrm{~F}_{5}\right)_{4} \cdot \mathrm{Et}_{2} \mathrm{O}$ (70 mg, $\left.0.1 \mathrm{mmol}\right)$ was added a $5 \mathrm{~mL}$ $\mathrm{CH}_{2} \mathrm{Cl}_{2}$ solution of $9 \mathbf{b}$ (100 $\left.\mathrm{mg}, 0.05 \mathrm{mmol}\right)$. The reaction mixture was stirred for $48 \mathrm{~h}$ under argon before filtration to remove $\mathrm{NaI}$. The filtrate was diluted with $\mathrm{CH}_{2} \mathrm{Cl}_{2}$ then washed with distilled water two times. The organic layer was dried over $\mathrm{MgSO}_{4}$. The solvent was removed under reduced pressure and the solid product was purified by recrystallization $\left(\mathrm{CHCl}_{3} /\right.$ methanol). Any remaining volatile components were removed under reduced pressure to afford a violet-black solid. Yield: $122 \mathrm{mg}$ (78\%). mp 65-66 ${ }^{\circ} \mathrm{C}$. ${ }^{1} \mathrm{H}$ NMR $\left(\mathrm{CDCl}_{3}\right): \delta$, ppm $9.32(\mathrm{~d}, 4 \mathrm{H}, J=$ $6.0 \mathrm{~Hz}$, pyridyl-H), 9.12 (d, 4H, $J=6 \mathrm{~Hz}$, pyridyl-H), 8.85 (d, 4H, $J=6.0 \mathrm{~Hz}, \beta$-pyrrole-H), 8.76 (d, 4H, $J=$ $3 \mathrm{~Hz}, \beta$-pyrrole-H), 7.41 (s, 4H, phenyl-H), 4.89 (s, 6H, $\left.\mathrm{N}-\mathrm{CH}_{3}\right), 4.32\left(\mathrm{t}, 4 \mathrm{H}, \mathrm{J}=6 \mathrm{~Hz}, \mathrm{O}-\mathrm{CH}_{2}-\right), 4.07(\mathrm{t}, 8 \mathrm{H}$, $\left.\mathrm{J}=6 \mathrm{~Hz}, \mathrm{O}-\mathrm{CH}_{2}^{-}\right), 2.01\left(\mathrm{~m}, 4 \mathrm{H}, \mathrm{O}-\mathrm{CH}_{2}-\mathrm{CH}_{2^{-}}\right), 1.80(\mathrm{~m}$, $\left.8 \mathrm{H}, \mathrm{O}-\mathrm{CH}_{2}-\mathrm{CH}_{2}^{-}\right), 1.76-1.18\left(\mathrm{~m}, 108 \mathrm{H},-\left(\mathrm{CH}_{2}\right)_{9}-\right), 0.84$ $\left(\mathrm{m}, 18 \mathrm{H},-\mathrm{CH}_{3}\right),-2.72(\mathrm{~s}, 2 \mathrm{H}, \mathrm{NH}) .{ }^{19} \mathrm{~F}$ NMR $\left(\mathrm{CDCl}_{3}\right)$ : $\delta$, ppm -132.7 (d, 2F), -162.3 (m, 1F), -166.5 (m, 2F). HR-MS (MALDI-TOF): $m / z 1752.3809\left[\mathrm{M}-2 \mathrm{~B}\left(\mathrm{C}_{6} \mathrm{~F}_{5}\right)_{4}+\right.$ $\mathrm{H}]^{+}, 1752.3881$ calcd. for $\mathrm{C}_{116} \mathrm{H}_{179} \mathrm{~N}_{6} \mathrm{O}_{6}$. UV-vis $\left(\mathrm{CH}_{2} \mathrm{Cl}_{2}\right)$ : $\lambda_{\max }, \mathrm{nm}\left(\varepsilon \times 10^{-3} \mathrm{M}^{-1} . \mathrm{cm}^{-1}\right) 445.0$ (153.3), 526.0 (11.9), 581.0 (16.1), 668.0 (10.1). Anal. calcd. for $\mathrm{C}_{164} \mathrm{H}_{178^{-}}$ $\mathrm{B}_{2} \mathrm{~F}_{40} \mathrm{~N}_{6} \mathrm{O}_{6}$ : $\mathrm{C}$ 63.32; H 5.77; N 2.70. Found: C 64.88; H $6.90 ; \mathrm{N} 3.25$.

5,15-di-(4- $N$-dodecanoylpyridinium-4-yl)-10,20bis[3,4,5-tris(dodecyloxy)phenyl]porphyrin dibromide (11). Porphyrin 4b $(200 \mathrm{mg}, 0.116 \mathrm{mmol})$ was added to $2.0 \mathrm{~g}(8 \mathrm{mmol})$ of 1-bromododecane in $100 \mathrm{~mL}$ of dry DMF and the mixture was then heated to reflux for $6 \mathrm{~h}$. After evaporation of the solvent, the residual solid was washed with ether and filtered out. Purification of the solid by chromatography on silica gel with $10 \% \mathrm{CH}_{3} \mathrm{OH}-$ $\mathrm{CH}_{2} \mathrm{Cl}_{2}$ gave the title product in $97 \%$ yield $(250 \mathrm{mg})$. mp
177-179 ${ }^{\circ} \mathrm{C} .{ }^{1} \mathrm{H}$ NMR $\left(\mathrm{CDCl}_{3}\right): \delta$, ppm $9.82(\mathrm{~d}, 4 \mathrm{H}, J=$ $6 \mathrm{~Hz}$, pyridyl-H), 9.03 (d, 4H, $J=6 \mathrm{~Hz}$, pyridyl-H), 8.85 (s, 4H, $\beta$-pyrrole- $\mathrm{H}$ ), 8.63 (d, 4H, $J=3 \mathrm{~Hz}, \beta$-pyrrole- $\mathrm{H}$ ), $7.32\left(\mathrm{~s}, 4 \mathrm{H}\right.$, phenyl-H), $5.34\left(\mathrm{t}, 4 \mathrm{H},{ }^{3} J_{\mathrm{HH}}=9 \mathrm{~Hz},{ }^{3} J_{\mathrm{HH}}=\right.$ $\left.6 \mathrm{~Hz}, \mathrm{~N}-\mathrm{CH}_{2^{-}}\right), 4.32$ (t, $\left.4 \mathrm{H}, \mathrm{J}=6 \mathrm{~Hz}, \mathrm{O}-\mathrm{CH}_{2}{ }^{-}\right), 4.09$ (t, $\left.8 \mathrm{H},{ }^{3} J_{\mathrm{HH}}=6 \mathrm{~Hz},{ }^{3} J_{\mathrm{HH}}=9 \mathrm{~Hz}, \mathrm{O}-\mathrm{CH}_{2}-\right), 2.39(\mathrm{~m}, 4 \mathrm{H}$, $\left.\mathrm{N}-\mathrm{CH}_{2}-\mathrm{CH}_{2}-\right), 1.88\left(\mathrm{~m}, 4 \mathrm{H}, \mathrm{O}-\mathrm{CH}_{2}-\mathrm{CH}_{2}-\right), 1.75(\mathrm{~m}, 8 \mathrm{H}$, $\left.\left.\mathrm{O}-\mathrm{CH}_{2}-\mathrm{CH}_{2}{ }^{-}\right), 1.53-1.22\left(\mathrm{~m}, 144 \mathrm{H},-\left(\mathrm{CH}_{2}\right)_{9}\right)^{-}\right), 0.85$ (m, $24 \mathrm{H},-\mathrm{CH}_{3}$ ), -3.55 (s, 2H, NH). MS (MALDI-TOF): $\mathrm{m} / \mathrm{z}$ $2060.76[\mathrm{M}-2 \mathrm{Br}+\mathrm{H}]^{+}, 2060.73$ calcd. for $\mathrm{C}_{138} \mathrm{H}_{223} \mathrm{~N}_{6} \mathrm{O}_{6}$. UV-vis $\left(\mathrm{CH}_{2} \mathrm{Cl}_{2}\right): \lambda_{\max }, \mathrm{nm}\left(\varepsilon \times 10^{-3} \mathrm{M}^{-1} . \mathrm{cm}^{-1}\right) 435.0$ (166), 526 (12), 575.0 (12), 663.0 (7.7). Anal. calcd. for $\mathrm{C}_{138} \mathrm{H}_{222} \mathrm{Br}_{2} \mathrm{~N}_{6} \mathrm{O}_{6}$ : C 74.62; H 10.07; N 3.78. Found: C 74.21; H 9.73; N 3.91 .

5,15-di-(4- $N$-dodecanoylpyridinium-4-yl)-10, 20-bis[3,4,5-tris(dodecyloxy)phenyl]porphyrin di-[tetrakis(pentafluorophenyl)borate] (12). To a $3 \mathrm{~mL}$ $\mathrm{CH}_{2} \mathrm{Cl}_{2}$ solution of $\mathrm{NaB}\left(\mathrm{C}_{6} \mathrm{~F}_{5}\right)_{4} \cdot \mathrm{Et}_{2} \mathrm{O}(68.4 \mathrm{mg}, 0.097 \mathrm{mmol})$ was added a $5 \mathrm{~mL}$ of $\mathrm{CH}_{2} \mathrm{Cl}_{2}$ solution of $\mathbf{1 1}(100 \mathrm{mg}$, $0.045 \mathrm{mmol})$. The reaction mixture was stirred overnight. The resulting solution was diluted with $\mathrm{CH}_{2} \mathrm{Cl}_{2}$ and washed with brine, distilled water and then dried over $\mathrm{MgSO}_{4}$. The organic solvent was removed under reduced pressure. The product was purified by recrystallization from $\mathrm{CHCl}_{3} /$ methanol. Any remaining volatile components were removed under reduced pressure to afford the violet-black solid (134 mg, yield: 87\%). mp 98-100 ${ }^{\circ} \mathrm{C}$. ${ }^{1} \mathrm{H}$ NMR $\left(\mathrm{CDCl}_{3}\right): \delta$, ppm $9.06(\mathrm{~d}, 4 \mathrm{H}, J=6 \mathrm{~Hz}$, pyridyl-H), 8.97 (d, 4H, $J=6 \mathrm{~Hz}, \beta$-pyrrole-H), 8.85 (d, 4H, $J=6 \mathrm{~Hz}, \beta$-pyrrole-H), $8.56(\mathrm{~d}, 4 \mathrm{H}, J=6 \mathrm{~Hz}$, pyridyl-H), $7.38\left(\mathrm{~s}, 4 \mathrm{H}\right.$, phenyl-H), $4.80\left(\mathrm{t}, 4 \mathrm{H},{ }^{3} J_{\mathrm{HH}}=9 \mathrm{~Hz},{ }^{3} J_{\mathrm{HH}}=\right.$ $\left.6 \mathrm{~Hz}, \mathrm{~N}-\mathrm{CH}_{2^{-}}\right), 4.27\left(\mathrm{t}, 4 \mathrm{H},{ }^{3} J_{\mathrm{HH}}=9 \mathrm{~Hz},{ }^{3} J_{\mathrm{HH}}=6 \mathrm{~Hz}\right.$, $\left.\mathrm{O}-\mathrm{CH}_{2^{-}}\right), 3.96\left(\mathrm{t}, 8 \mathrm{H}, \mathrm{J}=6 \mathrm{~Hz}, \mathrm{O}-\mathrm{CH}_{2^{-}}\right), 2.31(\mathrm{~m}, 4 \mathrm{H}$, $\left.\mathrm{N}-\mathrm{CH}_{2}-\mathrm{CH}_{2}-\right), 1.96\left(\mathrm{~m}, 4 \mathrm{H}, \mathrm{O}-\mathrm{CH}_{2}-\mathrm{CH}_{2}-\right), 1.67(\mathrm{~m}, 16 \mathrm{H}$, $\left.\mathrm{O}-\mathrm{CH}_{2}-\mathrm{CH}_{2}-\right), 1.50-1.18\left(\mathrm{~m}, 136 \mathrm{H},-\left(\mathrm{CH}_{2}\right)_{9}-\right), 0.88(\mathrm{~m}$, $\left.24 \mathrm{H},-\mathrm{CH}_{3}\right),-2.66(\mathrm{~s}, 2 \mathrm{H}, \mathrm{NH}) .{ }^{19} \mathrm{~F} \mathrm{NMR}\left(\mathrm{CDCl}_{3}\right): \delta$, ppm -132.6 (d, 2F), -162.2 (t, 1F), -166.3 (t, 2F). MS (MALDITOF): $m / z 2060.59\left[\mathrm{M}-2 \mathrm{~B}\left(\mathrm{C}_{6} \mathrm{~F}_{5}\right)_{4}+\mathrm{H}\right]^{+}, 2060.73$ calcd. for $\mathrm{C}_{138} \mathrm{H}_{223} \mathrm{~N}_{6} \mathrm{O}_{6}$. UV-vis $\left(\mathrm{CH}_{2} \mathrm{Cl}_{2}\right): \lambda_{\max }, \mathrm{nm}\left(\varepsilon \times 10^{-3}\right.$ $\left.\mathrm{M}^{-1} . \mathrm{cm}^{-1}\right) 453.0$ (153), 527.0 (12.7), 583.0 (19.4), 668.0 (12.7). Anal. calcd. for $\mathrm{C}_{186} \mathrm{H}_{222} \mathrm{~B}_{2} \mathrm{~F}_{40} \mathrm{~N}_{6} \mathrm{O}_{6}$ : C 65.33; H 6.54; N 2.46. Found: C 65.33; H 6.58; N 2.53.

\section{RESULTS AND DISCUSSION}

\section{Synthesis and characterization}

The synthetic approach in order to access to free base porphyrin precursors $\mathbf{3 a}-\mathbf{4 b}$ is shown in Scheme 1 . 4-(dodecyloxy)benzaldehyde and 3,4,5-tris(dodecyloxy)benzaldehyde were prepared according to the literature $[40,41]$. The free-base porphyrins $(\mathbf{3 a}, \mathbf{3 b}, \mathbf{4 a}, \mathbf{4 b})$ were successfully synthesized from the propionic acidcatalyzed condensation of 5-(4-pyridyl)dipyrromethane [42] with the corresponding aldehyde under reflux in the dark for $2.5 \mathrm{~h}$. The solvent was removed under reduced 


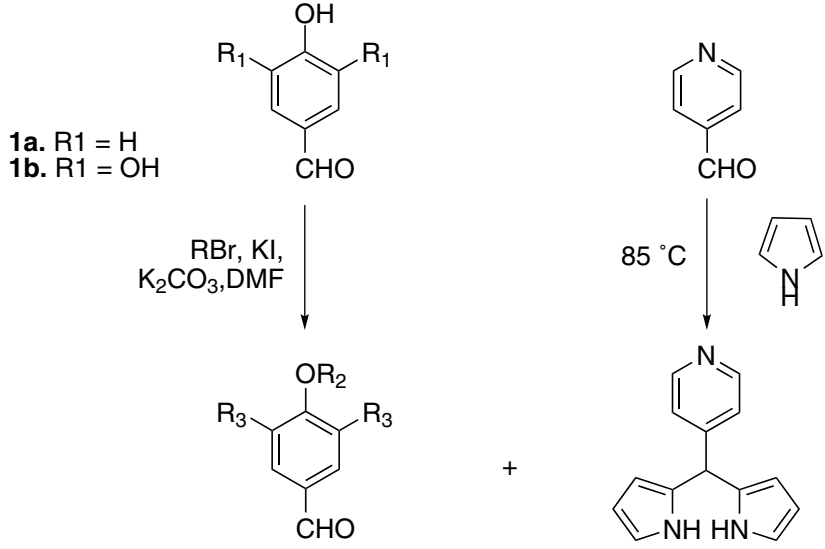

2a. $\mathrm{R}_{2}=\mathrm{C}_{12} \mathrm{H}_{25}, \mathrm{R}_{3}=\mathrm{H}$

2b. $\mathrm{R}_{2}=\mathrm{C}_{12} \mathrm{H}_{25}, \mathrm{R}_{3}=\mathrm{OC}_{12} \mathrm{H}_{25}$

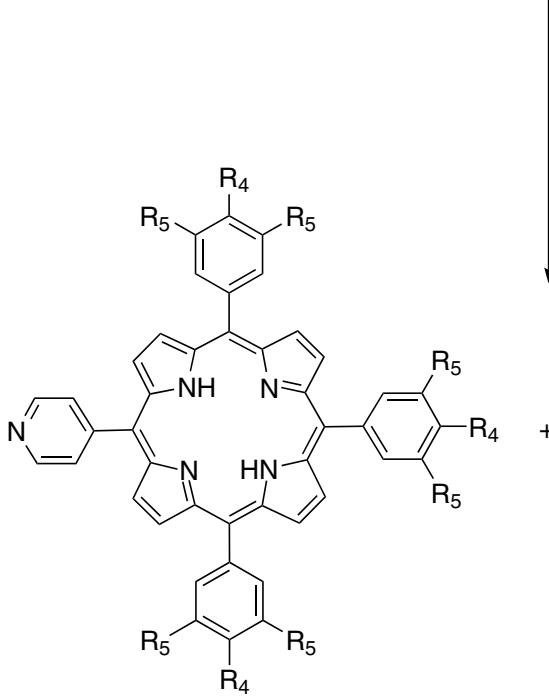

Propionic acid reflux

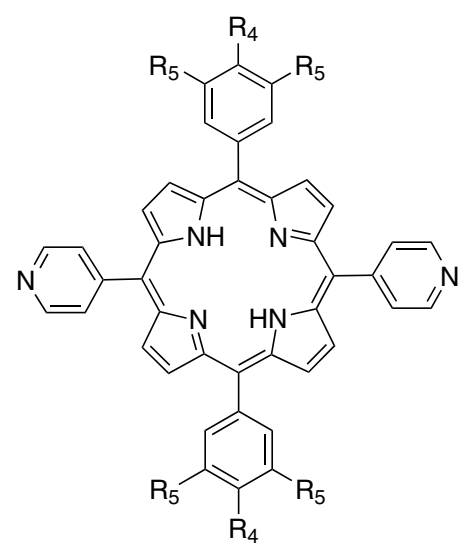

3a. $\mathrm{R}_{4}=\mathrm{OC}_{12} \mathrm{H}_{25}, \mathrm{R}_{5}=\mathrm{H}$

3b. $\mathrm{R}_{4}=\mathrm{R}_{5}=\mathrm{OC}_{12} \mathrm{H}_{25}$

4a. $\mathrm{R}_{4}=\mathrm{OC}_{12} \mathrm{H}_{25}, \mathrm{R}_{5}=\mathrm{H}$

4b. $\mathrm{R}_{4}=\mathrm{R}_{5}=\mathrm{OC}_{12} \mathrm{H}_{25}$ respectively. The phenyl and pyridyl meso substituents of the free-base porphyrin have signals located at 7.45 and $9.04 \mathrm{ppm}$, respectively. In the aliphatic area, relative to the long alkyl chains, two triplet signals at 4.33 (4H) and 4.12 $(8 \mathrm{H}) \mathrm{ppm}$ are respectively accounting for the methylene protons $\left(\alpha-\mathrm{CH}_{2}\right) 1^{\prime}$ and 1 linked to the oxygen atoms. The resonances of the $\beta$-methylene protons $2^{\prime}$ and 2 appear as muliplets at $2.01(4 \mathrm{H})$ and $1.90(8 \mathrm{H}) \mathrm{ppm}$, respectively. The methyl protons have signals located between $0.85-0.95 \mathrm{ppm}$. Other protons of the alkyl chains are less well resolved with resonances in the region 1.25-1.70 ppm. 3a, 3b and 4a exhibit similar proton NMR spectra as for 4b. The low-field signals (7.29-9.07 $\mathrm{ppm}$ ) correspond to the protons of the pyridyl groups, $\beta$-pyrrole and phenyl groups. The high-field signals (singlet at about $-2.80 \mathrm{ppm}, 2 \mathrm{H}$ ) are assigned to the $\mathrm{NH}$ protons. The resonances for the $\alpha$-methylene and $\beta$-methylene protons appear at around 4.11 and $2.01 \mathrm{ppm}$ as triplets, respectively. The resonances of the remaining protons of the methylene and methyl groups are located between 0.91 and $2.06 \mathrm{ppm}$. ${ }^{1} \mathrm{H}$ NMR data of these derivatives are given in the Experimental Section and spectra are shown in the Supporting information.

The synthetic routes used for the preparation of the ionic porphyrin derivatives are shown in Schemes 2 and 3.

Scheme 1. Synthetic scheme of porphyrins 3 and 4

The bromide salts ( $\mathbf{7}$ and $\mathbf{1 1}$ ) were synthesized by reaction of 1-bromododecane with the corresponding neutral porphyrin in DMF at $70{ }^{\circ} \mathrm{C}$ followed by purification by column chromatography and recrystallization in nearly quantitative yields. Similarly, compounds 3 and $\mathbf{4}$ were methylated in a dimethylformamide/chloroform mixture under reflux by using $\mathrm{CH}_{3} \mathrm{I}$ to afford compounds 5 and 9 in 97\% yield (Schemes 2 and 3). Tetrakis(perfluorophenyl)borate salts were prepared by anionic exchange of bromide or iodide porphyrins with a little excess of sodium tetrakis(perfluorophenyl)borate $\left(\mathrm{NaB}\left(\mathrm{C}_{6} \mathrm{~F}_{5}\right)_{4}\right)$ in dichloromethane at room temperature. The insoluble, inorganic salts, $\mathrm{NaBr}$ and $\mathrm{NaI}$, were removed by filtration under vacuum and the organic solutions were washed with distilled water. The porphyrin salts were finally isolated in relatively high yields (69-87\%). However, the reaction of 9a with sodium tetrakis(perfluorophenyl)borate was not successful using the similar synthetic method described for $9 \mathbf{b}$, probably due to the poor solubility of the precursor 9a in the organic solvent. All these porphyrin salts which are assigned to the $\beta$-pyrrole and $\mathrm{NH}$ protons 


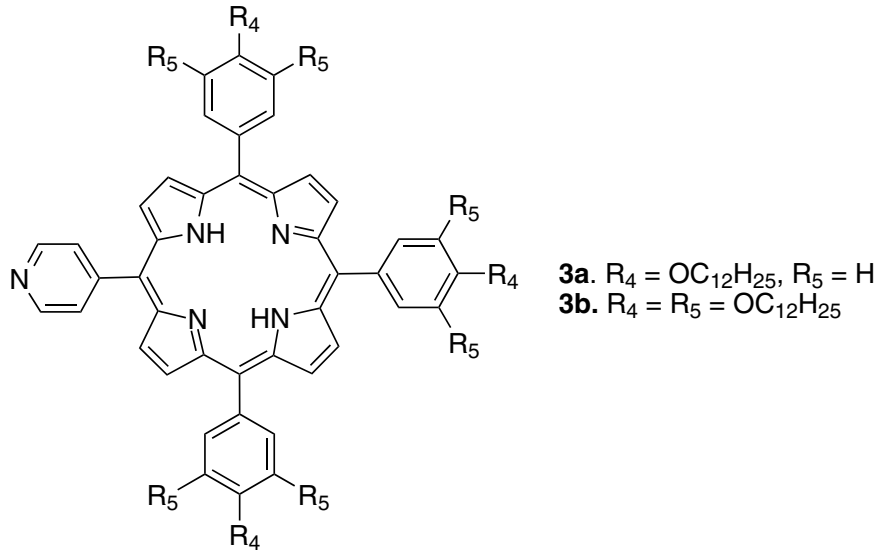

. $\mathrm{OC}_{12} \mathrm{H}_{25}, \mathrm{R}_{5}=\mathrm{H}$
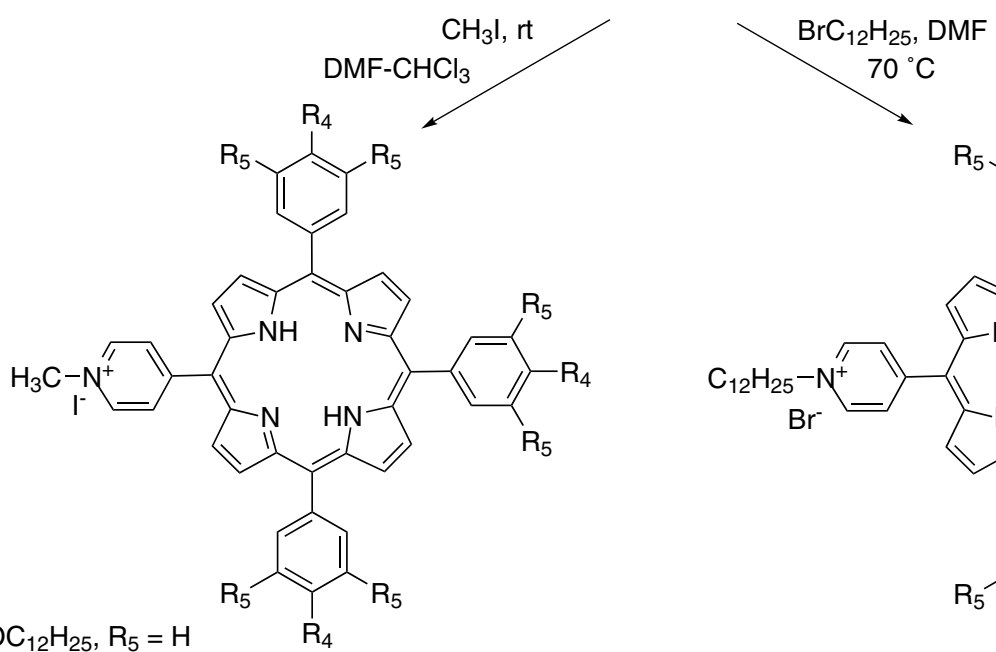

5b. $\mathrm{R}_{4}=\mathrm{R}_{5}=\mathrm{OC}_{12} \mathrm{H}_{25}$

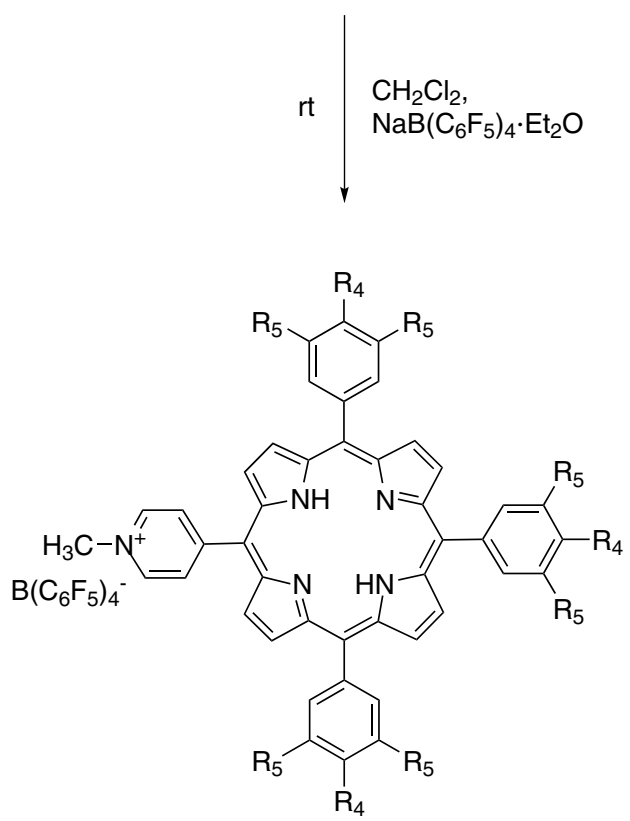

6a. $\mathrm{R}_{4}=\mathrm{OC}_{12} \mathrm{H}_{25}, \mathrm{R}_{5}=\mathrm{H}$

6b. $\mathrm{R}_{4}=\mathrm{R}_{5}=\mathrm{OC}_{12} \mathrm{H}_{25}$

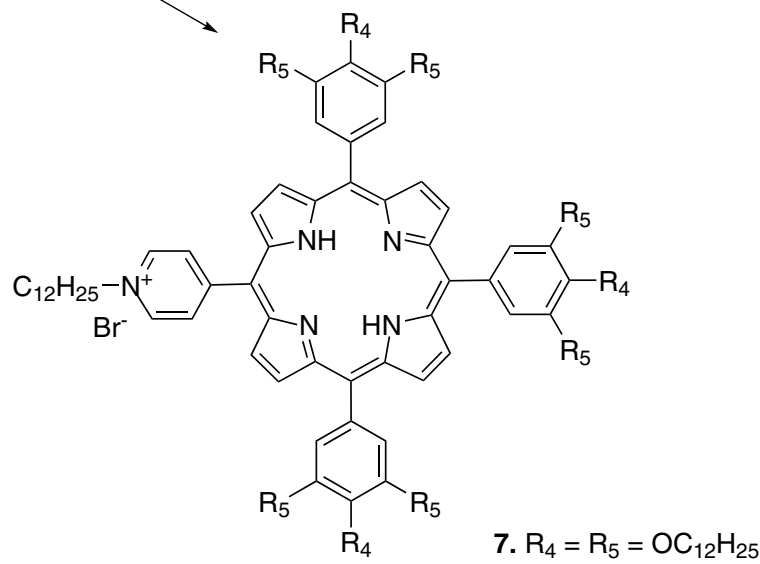

$$
\begin{aligned}
& \mathrm{CH}_{2} \mathrm{Cl}_{2}, \\
& \mathrm{NaB}\left(\mathrm{C}_{6} \mathrm{~F}_{5}\right)_{4} \cdot \mathrm{Et}_{2} \mathrm{O}
\end{aligned}
$$

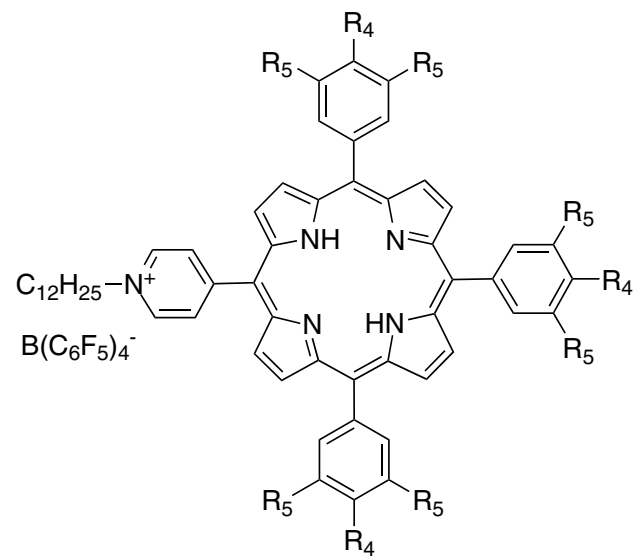

8. $\mathrm{R}_{4}=\mathrm{R}_{5}=\mathrm{OC}_{12} \mathrm{H}_{25}$

Scheme 2. Synthetic scheme of cationic porphyrins $\mathbf{5 a}-\mathbf{8}$ 


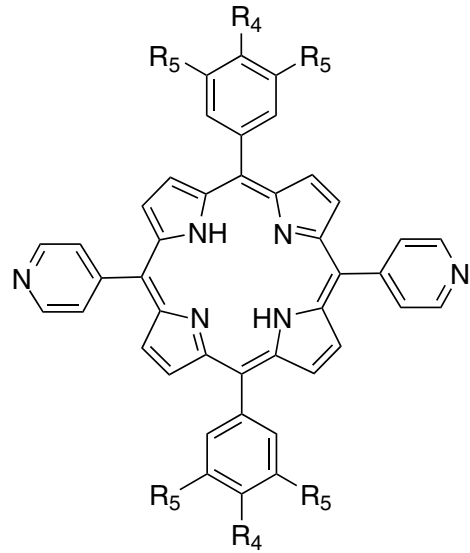

4a. $\mathrm{R}_{4}=\mathrm{OC}_{12} \mathrm{H}_{25}, \mathrm{R}_{5}=\mathrm{H}$

4b. $\mathrm{R}_{4}=\mathrm{R}_{5}=\mathrm{OC}_{12} \mathrm{H}_{25}$

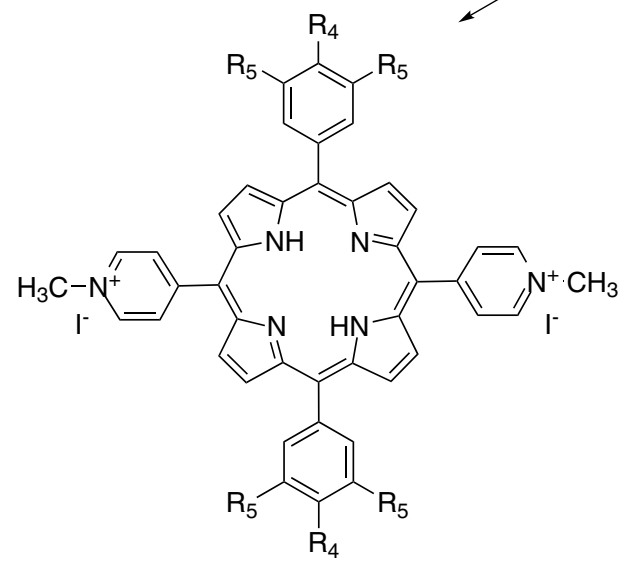

9a. $\mathrm{R}_{4}=\mathrm{OC}_{12} \mathrm{H}_{25}, \mathrm{R}_{5}=\mathrm{H}$

9b. $\mathrm{R}_{4}=\mathrm{R}_{5}=\mathrm{OC}_{12} \mathrm{H}_{25}$

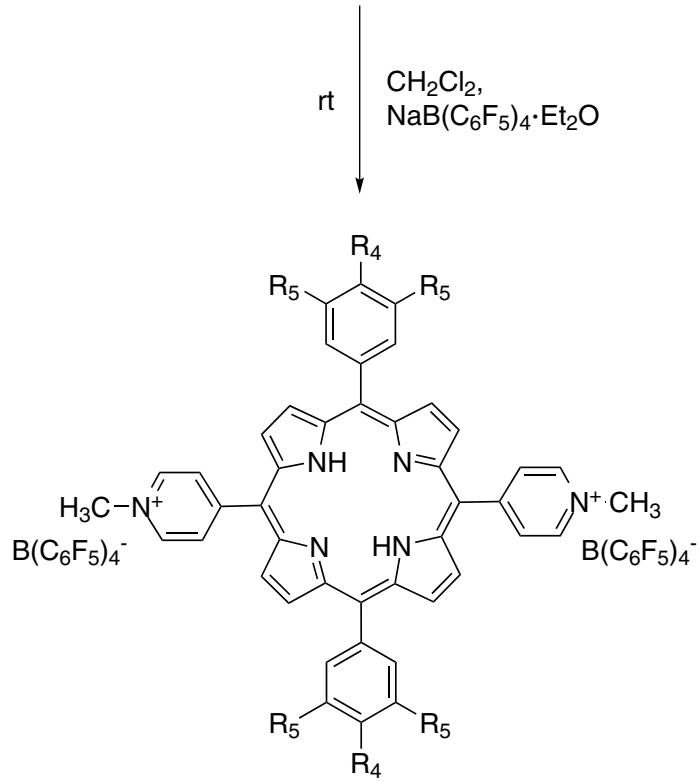

10. $\mathrm{R}_{4}=\mathrm{R}_{5}=\mathrm{OC}_{12} \mathrm{H}_{25}$

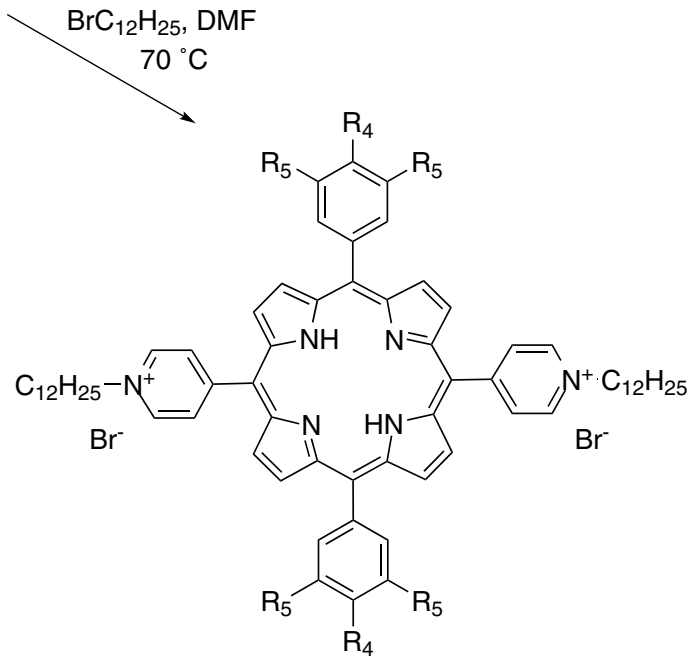

11. $\mathrm{R}_{4}=\mathrm{R}_{5}=\mathrm{OC}_{12} \mathrm{H}_{25}$

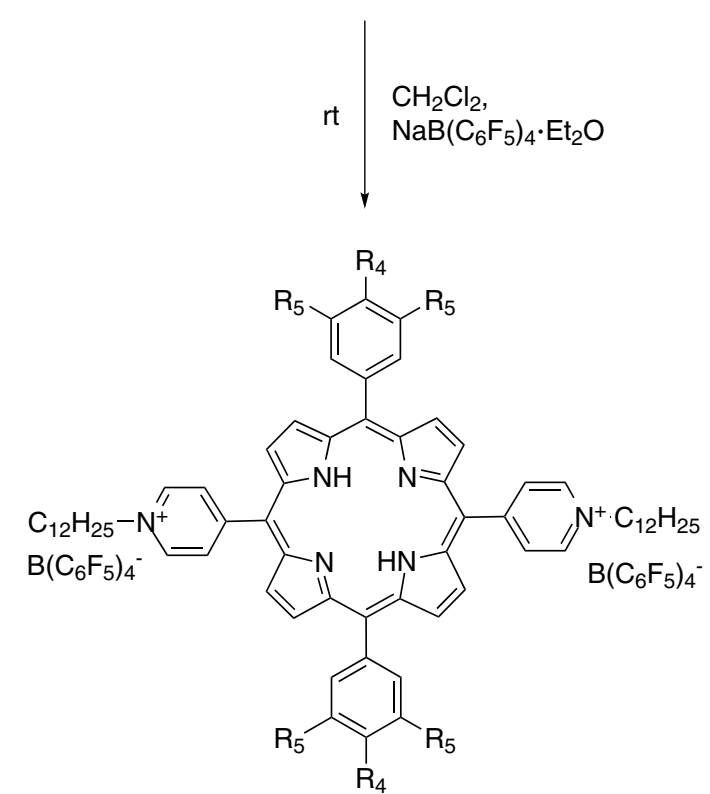

12. $\mathrm{R}_{4}=\mathrm{R}_{5}=\mathrm{OC}_{12} \mathrm{H}_{25}$

Scheme 3. Synthetic scheme of cationic porphyrins $\mathbf{9 a - 1 2}$ 


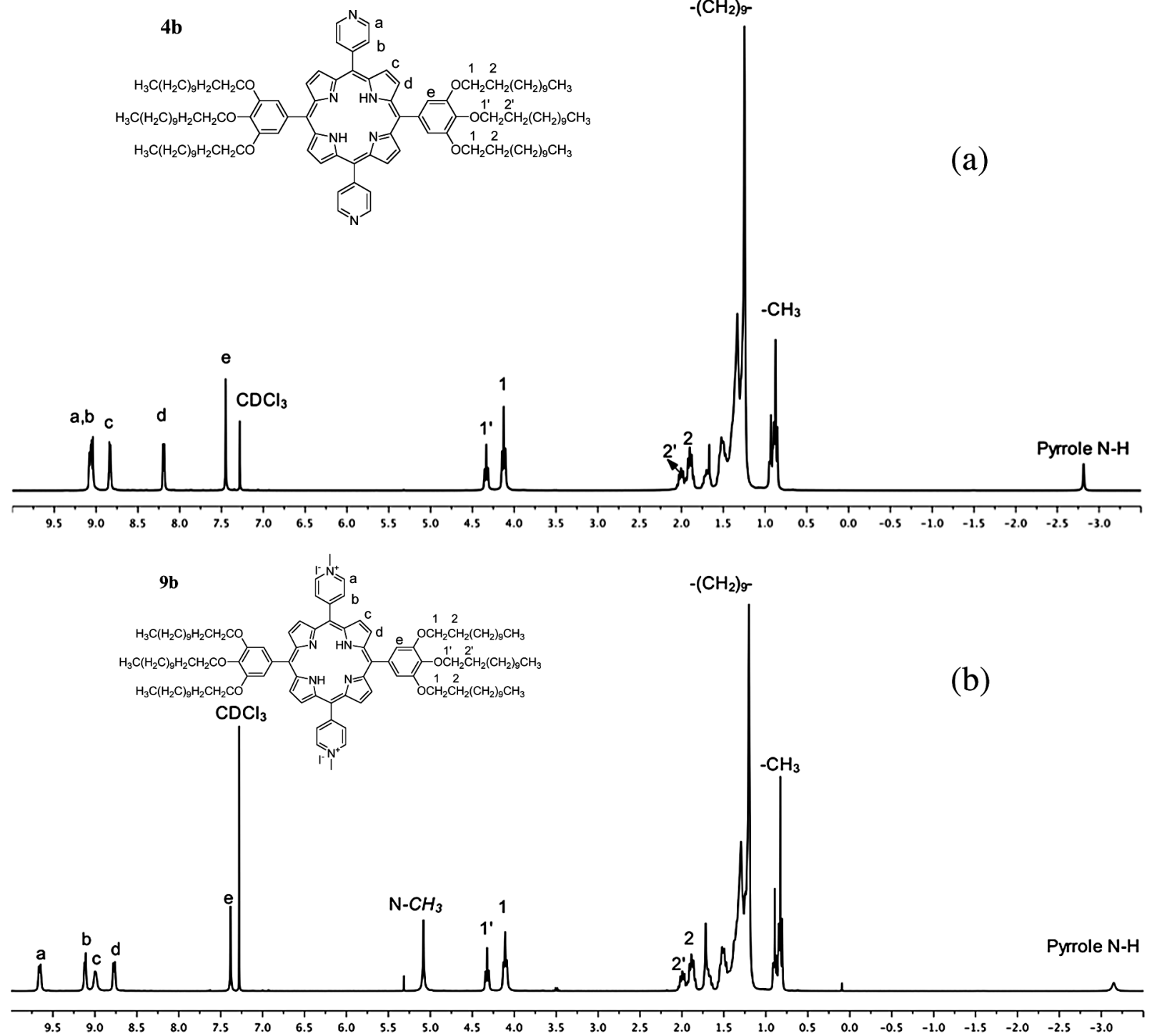

$-\left(\mathrm{CH}_{2}\right) 9$

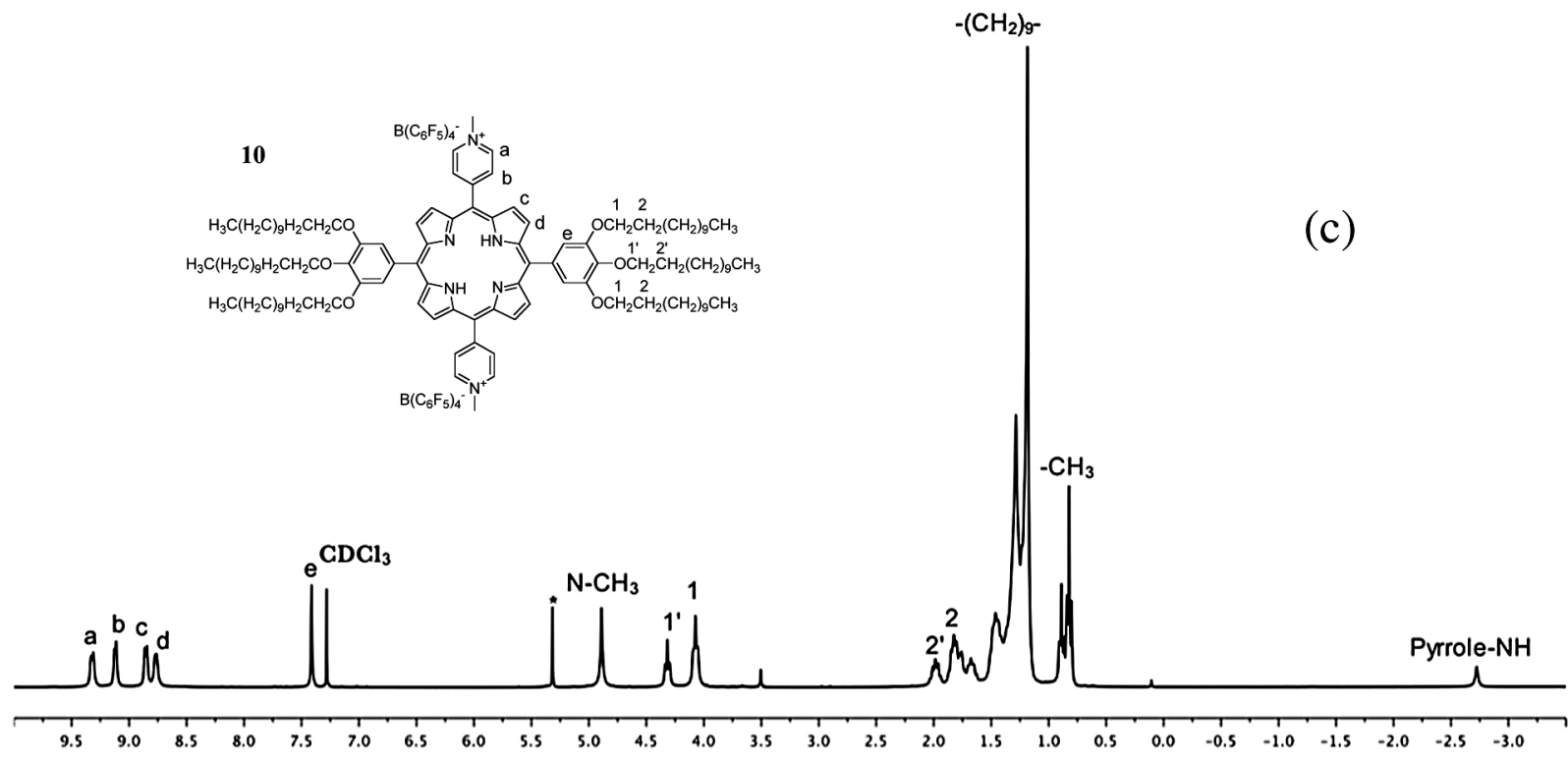

\section{$-\left(\mathrm{CH}_{2}\right)_{9}-$}

Fig. 1. Representative $300 \mathrm{MHz}{ }^{1} \mathrm{H}$ NMR spectra of porphyrins $\mathbf{4 b}$ (a), $9 \mathbf{b}$ (b) and $\mathbf{1 0}$ (c) in $\mathrm{CDCl}_{3}$ ("solvent: $\mathrm{CH}_{2} \mathrm{Cl}_{2}$ ) 
are very immiscible with water and the hydrophobicity of these compounds can be attributed to the high waterrepellency of the long alkyl chains of the cationic part of the molecule and the low polarizability. This property facilitated purification by simply washing the crude product with distilled water in order to remove any traces of water soluble impurities.

Some of the porphyrin salts that have been prepared are solid with exception of compounds $\mathbf{5 b}, \mathbf{6 a}$, and $\mathbf{8}$ which are liquid at room temperature. All the structures and compositions of these new porphyrin salts were determined by ${ }^{1} \mathrm{H}$ and ${ }^{19} \mathrm{~F}$ NMR spectroscopy, MALDITOF mass spectrometry (MS and HRMS), UV-visible spectroscopy and elemental analysis. MALDI-TOF mass spectrometry technique was used firstly to characterize these porphyrin compounds and their salts. All the studied compounds exhibit one main peak (protonated molecule for neutral derivatives or cationic part of the molecule for ionic liquids). These data confirmed the expected structures. For example, the MALDI-TOF mass spectrum of 8 exhibits the parent-ion peak at $\mathrm{m} / z=2442.00$ for cationic part (calculated for $\mathrm{C}_{163} \mathrm{H}_{270} \mathrm{~N}_{5} \mathrm{O}_{9} \mathrm{~m} / \mathrm{z}=2442.08$ ) (Fig. 2).

The well-resolved proton NMR spectra of $\mathbf{5 a}, \mathbf{5 b}, \mathbf{7}$, 9a, 9b and 11, shown in the Supporting information are very well consistent with the expected structures and exhibit similar chemical shifts. The proton resonances occur in four distinct regions. The downfield region from 7 to $c a$. $10 \mathrm{ppm}$ correspond to pyridine ring protons ( $c a$. 9.80 and 9.03 ppm for 7 and 11, ca. 9.67 and $9.12 \mathrm{ppm}$ for $\mathbf{5 b}$ and $\mathbf{9 b}, c a .9 .44$ and $9.00 \mathrm{ppm}$ for $\mathbf{5 a}$ and $\mathbf{9 a}$ ), $\beta$-pyrrolic protons (ca. 9.00 and 8.70 ppm for $\mathbf{7 , 1 1}, \mathbf{5 b}$ and $\mathbf{9 b} ; 8.86$ and $8.63 \mathrm{ppm}$ for $\mathbf{5 a}, 9.01 \mathrm{ppm}$ for $\mathbf{9 a}$ ) and phenyl protons ( $c a .7 .30 \mathrm{ppm}$ for $\mathbf{9 b}$ and $\mathbf{1 1}$ as singlets, $7.43 \mathrm{ppm}$ for $\mathbf{5 b}$ and $\mathbf{7}$ as singlets, $7.09-8.08 \mathrm{ppm}$ for 5a as doublets, 8.11 and $7.40 \mathrm{ppm}$ for $\mathbf{9 a}$ as doublets). The medium range resonances (between 4 and $6 \mathrm{ppm}$ ) are respectively attributed to $-\mathrm{O}-\mathrm{CH}_{2}$-protons $(4.27 \mathrm{ppm}$ for $\mathbf{9 a} ; 4.26$ and $4.11 \mathrm{ppm}$ for $\mathbf{5 a} ; 4.33$ and $4.12 \mathrm{ppm}$ for $\mathbf{5 b}, \mathbf{7}, \mathbf{9 b}$ and $\mathbf{1 1}$ ), $\mathrm{N}-\mathrm{CH}_{2}$-protons (5.37 ppm for 7; $5.34 \mathrm{ppm}$ for 11) and $\mathrm{N}-\mathrm{CH}_{3}$ protons (ca. $5.10 \mathrm{ppm}$ for $\mathbf{5 b}$ and $\mathbf{9 b} ; 4.93$ ppm for $\mathbf{5 a} ; 4.72 \mathrm{ppm}$ for $\mathbf{9 a})$. Higher field resonances (between 0.5 and $2.5 \mathrm{ppm}$ ) are attributed to the remaining aliphatic protons. The last region near $-3.0 \mathrm{ppm}$ corresponds to inner $\mathrm{NH}$ proton resonances (-2.70 ppm for $\mathbf{5 b}$ and $\mathbf{7} ;-2.74 \mathrm{ppm}$ for $\mathbf{5 a} ;-2.94 \mathrm{ppm}$ for $9 \mathbf{a} ;-3.62 \mathrm{ppm}$ for $\mathbf{1 1} ;-3.15 \mathrm{ppm}$ for $\mathbf{9 b})$. By comparison of the proton NMR spectra of the ionic porphyrins with that of the corresponding neutral ones, the alkylated pyridyl rings do not induce significant shifts on proton resonances with the exception of the pyridine $\alpha$ protons which demonstrate a downfield shift. As an example, the ${ }^{1} \mathrm{H}$ NMR spectrum of $\mathbf{9 b}$ is given on Fig. 1b. For $\mathbf{9 b}$, pyridine $\alpha$ protons are shifted to $9.66 \mathrm{pm}(9.04 \mathrm{ppm}$ for 4b) while pyridine $\alpha$ protons resonances remain almost unchanged (see Fig. 1a for comparison).

The completion of the anionic exchange reaction of bromide or iodide with tetrakis(perfluorophenyl)borate was confirmed by mass spectrometry, ${ }^{1} \mathrm{H}$ NMR and ${ }^{19} \mathrm{~F}$ NMR. In the negative mode MALDI/TOF mass spectra of these derivatives, a single ionic pattern a $\mathrm{m} / \mathrm{z}=679$ corresponding to the $\mathrm{B}\left(\mathrm{C}_{6} \mathrm{~F}_{5}\right)_{4}{ }^{-}$ion was detected. In contrast to the precursors, remarkable changes in the proton NMR spectra take place in the pyridinium $\alpha$ protons, the

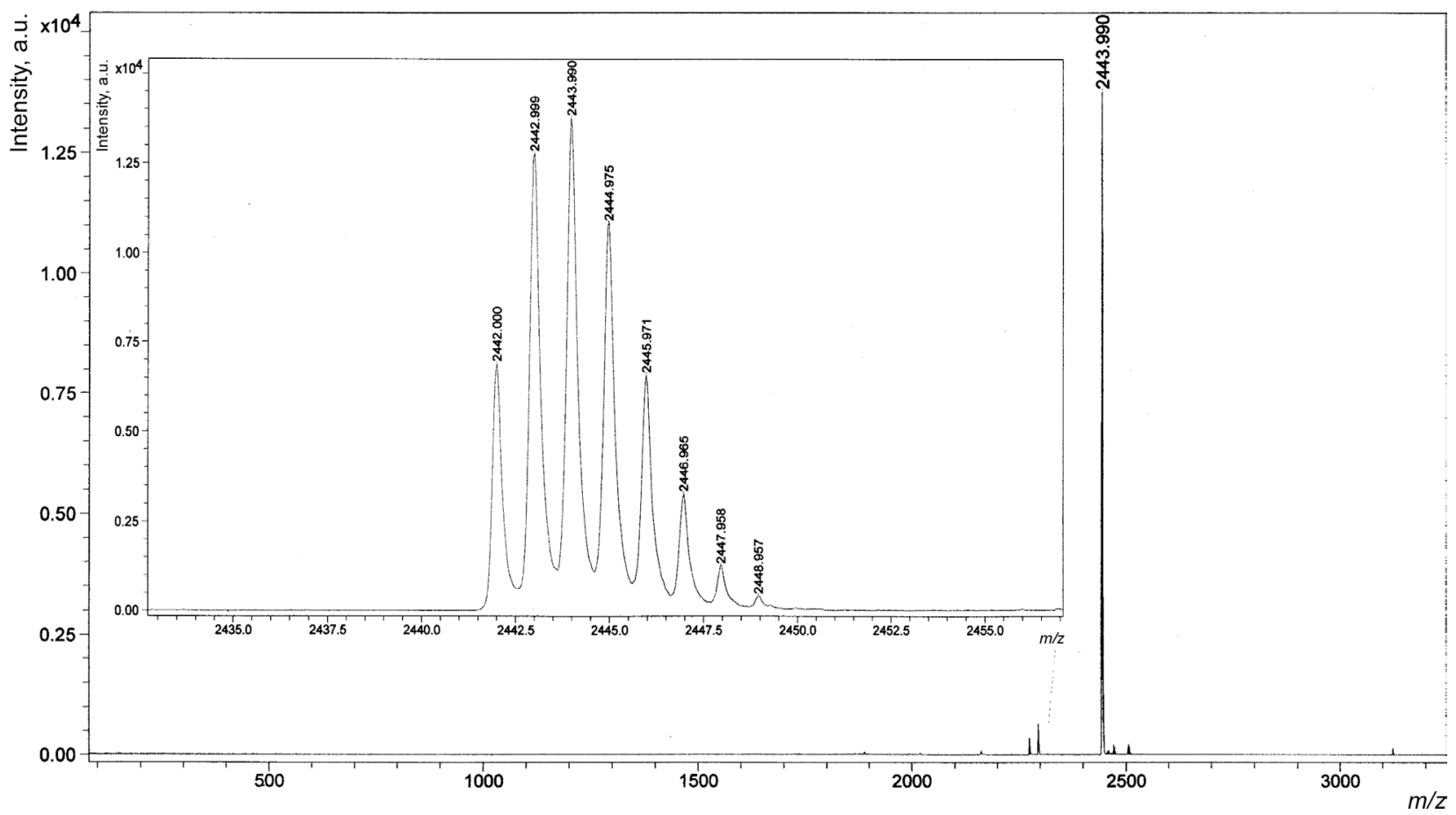

Fig. 2. MALDI-TOF mass spectrum of $\mathbf{8}$ (cationic part) 
methylene or the methyl groups adjacent to $\mathrm{N}$-pyridyl $\left(\mathrm{N}-\mathrm{CH}_{2}-\right)$ or $\left(\mathrm{N}-\mathrm{CH}_{3}\right)$ which are shifted to higher field due to the influence of the large $\mathrm{B}\left(\mathrm{C}_{6} \mathrm{~F}_{5}\right)_{4}{ }_{4}$ anion. The remaining proton resonances do not exhibit change with respect to the precursors. As an example, the ${ }^{1} \mathrm{H}$ NMR spectrum of $\mathbf{1 0}$ is given in Fig. 1c. When compared to the spectrum of $\mathbf{9 b}$ (Fig. 1b), the pyridinium $\alpha$ and $\mathrm{N}-\mathrm{CH}_{3}$ protons of 10 appear at 9.32 and $4.32 \mathrm{ppm}$, respectively (9.66 and $5.08 \mathrm{ppm}$ for $\mathbf{9 b}$ respectively). In addition, the resonances of the inner $\mathbf{~ N H}$ protons in $\mathbf{1 0}$ are shifted to lower field $(-2.72 \mathrm{ppm}$ compared to $\mathbf{9 b}(-3.15 \mathrm{ppm}))$. The ${ }^{19} \mathrm{~F}$ NMR spectra (given in Supporting information) of $\mathbf{6 a}, \mathbf{6 b}, \mathbf{8 a}, 10$

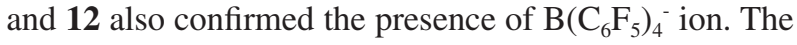
resonances of the para-, ortho- and meta-fluorine atoms appear at $-162.2,-132.6$ and $-166.3 \mathrm{ppm}$ respectively.

The UV-vis data of the neutral porphyrins and the porphyrin salts are given in Table 1 . The UV-vis spectra of $\mathbf{4 b}, 9 \mathbf{b}$ and 10 in $\mathrm{CH}_{2} \mathrm{Cl}_{2}$ are presented in Fig. 3. The neutral porphyrin $\mathbf{4 b}$ shows a typical electronic spectrum of meso-substituted porphyrins in $\mathrm{CH}_{2} \mathrm{Cl}_{2}$ with a sharp Soret band at $421 \mathrm{~nm}$ and four Q-bands at 519, 550, 591 and $646 \mathrm{~nm}$. Upon cationization of the neutral porphyrin $\mathbf{4 b}$, the number of absorption bands in $\mathbf{9 b}$ and $\mathbf{1 0}$ is reduced. Three Q-bands appear at 526 (526), 575 (581) and 663 (668) $\mathrm{nm}$ for $\mathbf{9 b}(\mathbf{1 0})$. The most relevant feature is the red-shift of the Soret band upon alkylation of the pyridyl groups. For example, the Soret band of the $N$-methylated porphyrin $\mathbf{9 b}$ is red-shifted by $20 \mathrm{~nm}$ from the corresponding Soret bands of $\mathbf{4 b}$. When $\mathrm{I}^{-}$is substituted by $\mathrm{B}\left(\mathrm{C}_{6} \mathrm{~F}_{5}\right)_{4}{ }_{4}$, the Soret band of $\mathbf{1 0}$ is further red-shifted to $445 \mathrm{~nm}$ (see Fig. 3). Similar trend is observed for other derivatives (Table 1). Another interesting feature can be observed on the UV-vis spectra. The Soret bands of

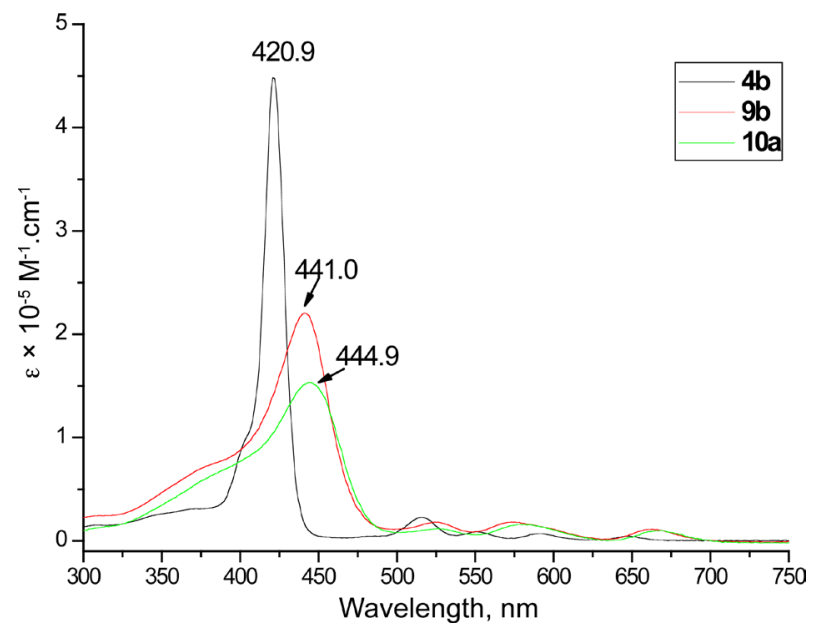

Fig. 3. UV-vis spectrum $\left(\mathrm{CH}_{2} \mathrm{Cl}_{2}\right)$ of porphyrins $\mathbf{4 b}, \mathbf{9 b}$ and $\mathbf{1 0}$

the porphyrin salts are significantly broadened and their extinction coefficients are remarkably decreased relative to those of the corresponding neutral porphyrins (Fig. 3 and Table 1).

\section{TGA measurements}

TGA analyses were performed under a $\mathrm{Ar} / \mathrm{O}_{2}$ gas stream mixture for six porphyrins in order to correlate their thermal stability to their structure (Fig. 4). The less stable structures are porphyrins $\mathbf{9 b}$ and $5 \mathbf{a}$ since their decomposition began at $183^{\circ} \mathrm{C}$ and $193{ }^{\circ} \mathrm{C}$ respectively and a further mass decrease occurred starting from $370{ }^{\circ} \mathrm{C}$ due to the porphyrin calcination. TGA curves indicated a first $13.5 \%$ weight loss for $\mathbf{9 b}$ and $11.3 \%$ for 5a assigned to methyl iodide release during the heating

Table 1. UV-vis absorption data in $\mathrm{CH}_{2} \mathrm{Cl}_{2}$ for the synthesized derivatives

\begin{tabular}{|c|c|c|c|c|c|}
\hline \multirow{3}{*}{$\begin{array}{l}\text { Compound } \\
\mathbf{3 a}\end{array}$} & \multicolumn{5}{|c|}{$\lambda_{\max }, \mathrm{nm}\left(\varepsilon \times 10^{-3}, \mathrm{M}^{-1} \cdot \mathrm{cm}^{-1}\right)$} \\
\hline & \multicolumn{2}{|c|}{ Soret band } & \multicolumn{3}{|c|}{ Q-bands } \\
\hline & 421 (1769) & $517(71)$ & $555(46)$ & $592(26)$ & $650(27)$ \\
\hline $3 \mathbf{b}$ & $421(350)$ & $516(18)$ & $552(8.7)$ & $591(4.8)$ & $649(4.2)$ \\
\hline $4 \mathbf{a}$ & $420(1622)$ & $515(72)$ & $552(37)$ & $591(23)$ & $650(18)$ \\
\hline $4 b$ & 421 (449) & $516(22.7)$ & $550(8.8)$ & $591(7.0)$ & 646 (4.6) \\
\hline $5 \mathbf{a}$ & $422(143)$ & $522(11)$ & $584(13)$ & $663(0.8)$ & \\
\hline $5 b$ & 428 (122) & $522(12)$ & $589(1.1)$ & $659(0.6)$ & \\
\hline $\mathbf{6 a}$ & 419 (131) & 590 (1.6) & $661(1.1)$ & & \\
\hline $6 \mathbf{b}$ & 421 (113) & 589 (14) & $633(0.8)$ & & \\
\hline 7 & $427(131)$ & $524(13)$ & $593(13)$ & $659(0.7)$ & \\
\hline 8 & 423 (118) & $593(15)$ & $664(0.9)$ & & \\
\hline $9 a$ & 438 (115) & $526(9.4)$ & $574(11)$ & $666(7.5)$ & \\
\hline $9 b$ & $441(220)$ & $526(17.9)$ & $575(18.1)$ & $663(11.1)$ & \\
\hline 10 & 445 (153) & $526(11.9)$ & $581(16.1)$ & $668(10.1)$ & \\
\hline 11 & 435 (166) & $526(12)$ & $575(12)$ & $663(7.7)$ & \\
\hline 12 & 453 (153) & $527(12.7)$ & $581(19.4)$ & 668 (12.7) & \\
\hline
\end{tabular}




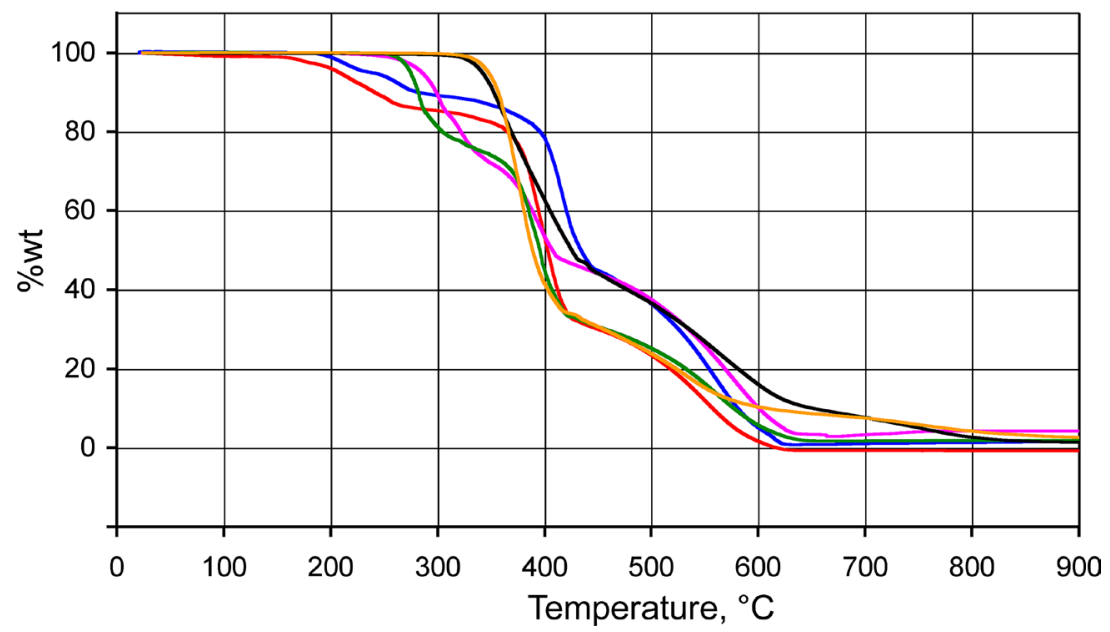

Fig. 4. TGA traces for porphyrins $5 \mathbf{a}$ (blue line), 6a (black line), 9a (pink line), 9b (red line), 11 (green line) and $\mathbf{1 2}$ (orange line) under an $\mathrm{Ar} / \mathrm{O}_{2}$ gas stream $\left(30 \mathrm{~mL} \cdot \mathrm{min}^{-1} / 10 \mathrm{~mL} \cdot \mathrm{min}^{-1}\right)$

process. The respective theoretical values are $14.1 \%$ and $10.8 \%$. This result demonstrates that when iodide is used as the counter-anion, the thermal stability is quite low compared to porphyrins 11,12 and $\mathbf{6 a}$ with other counter-anions. Compound $\mathbf{9 a}$ is surprisingly more stable than $\mathbf{9 b}$ and $\mathbf{5 a}$ despite the presence of two iodide ions and this is may be due to electronic effects since 9a possess less donor atoms and is then less oxidizable. Nevertheless, a straightforward correlation was observed between the thermal stability and the nature of the counter-anion if we compared $11 v s . \mathbf{1 2}, \mathbf{5 a} v s .6 \mathbf{a}$ and $9 \mathbf{b} v s$. 11. From this result, a stability series clearly emerged: $\mathrm{I}^{-}<\mathrm{Br}^{-}<<$ $\mathrm{B}\left(\mathrm{C}_{6} \mathrm{~F}_{5}\right)_{4}$. Moreover, if we compare $\mathbf{6 a}$ and $\mathbf{1 2}$, the number and the length of the peripheral alkyl chains seem to have no effect on the thermal stability of the porphyrins.

\section{Phase transition}

The thermal properties and solid-liquid phase transitions of these porphyrin salts were examined by differential scanning calorimetry (DSC) upon heating and cooling down (heating and cooling rate: $10{ }^{\circ} \mathrm{C}$. $\left.\mathrm{min}^{-1}\right)$. Endothermic or exothermic peaks assigned as the melting or crystallizing processes were observed below $300{ }^{\circ} \mathrm{C}$. The measured data of glass transition temperature ( $\mathrm{Tg}$ ), solid-solid transition (Ts-s), and melting point (Tm) are presented in Table 2. Porphyrin 9a has a melting point at $236{ }^{\circ} \mathrm{C} . \mathbf{5 a}, \mathbf{6 a}, 7$ and $9 \mathbf{b}$ have melting points below $200{ }^{\circ} \mathrm{C}$, while $\mathbf{5 b}, \mathbf{6 b}, \mathbf{8}, \mathbf{1 0}$ and $\mathbf{1 2}$ have melting temperatures ranging from 98 to $181{ }^{\circ} \mathrm{C}$. More interesting is the behavior of $\mathbf{5 b}, \mathbf{6 b}$, and $\mathbf{8}$ salts which can be considered as room-temperature ionic liquids due to low melting points observed below $25^{\circ} \mathrm{C}$. To our knowledge, these are the first examples of porphyrins displaying ionic liquid properties close to room temperature.

The first type of behavior is characterized by the salts that have a melting transition $(\mathbf{6 a}, 6 \mathbf{6}, \mathbf{7}, 9 \mathbf{b}, \mathbf{1 0}, \mathbf{1 1}$ and 12 in Table 1). The second type of behavior is represented by a number of salts that exhibited one solid-solid

Table 2. Thermal and conductivity properties of the ionic liquid salts

\begin{tabular}{|c|c|c|c|c|c|c|}
\hline Compound & $\mathrm{Tg},{ }^{\circ} \mathrm{C}^{\mathrm{a}}$ & Ts-s, ${ }^{\circ} \mathrm{C}^{\mathrm{b}}$ & $\mathrm{Tm},{ }^{\circ} \mathrm{C}^{\mathrm{c}}$ & $\Delta \mathrm{Hm}, \mathrm{kJ} \cdot \mathrm{mol}^{-1}$ & $\Delta \mathrm{Sm}, \mathrm{J}^{\mathrm{mol}}{ }^{-1} \cdot \mathrm{K}^{-1 \mathrm{~d}}$ & $\kappa / \mathrm{mS} \mathrm{cm}^{-1 \mathrm{e}}$ \\
\hline $5 \mathbf{a}$ & & 91 & 181 & 26.61 & 58.09 & solid \\
\hline $5 \mathbf{b}$ & -28 & & & & & 1.32 \\
\hline $6 \mathbf{a}$ & & & 129 & 67.30 & 163.35 & solid \\
\hline $6 b$ & & & -23 & 8.53 & 34.13 & 4.01 \\
\hline 7 & & & 145 & 17.99 & 42.75 & solid \\
\hline 8 & & -39 & -22 & 1.28 & 5.12 & 0.05 \\
\hline $9 a$ & & 213 & 236 & 27.86 & 54.00 & solid \\
\hline $9 b$ & & & 181 & 37.18 & 81.89 & solid \\
\hline 10 & & & 66 & 21.43 & 49.72 & solid \\
\hline 11 & & & 177 & 31.73 & 70.52 & solid \\
\hline 12 & & & 98 & 88.35 & 236.24 & solid \\
\hline
\end{tabular}

${ }^{\mathrm{a}}$ Glass transition. ${ }^{\mathrm{b}}$ Solid-solid transition. ${ }^{\mathrm{c}}$ Melting point. ${ }^{\mathrm{d}}$ Entropy of fusion $(\Delta \mathrm{Sm}=\Delta \mathrm{Hm} / \mathrm{Tm}$, where $\Delta \mathrm{Hm}$ is melting enthalpy at $\mathrm{Tm}(\mathrm{K}))$. ${ }^{\mathrm{e}}$ Specific conductivity at $22{ }^{\circ} \mathrm{C}$. 


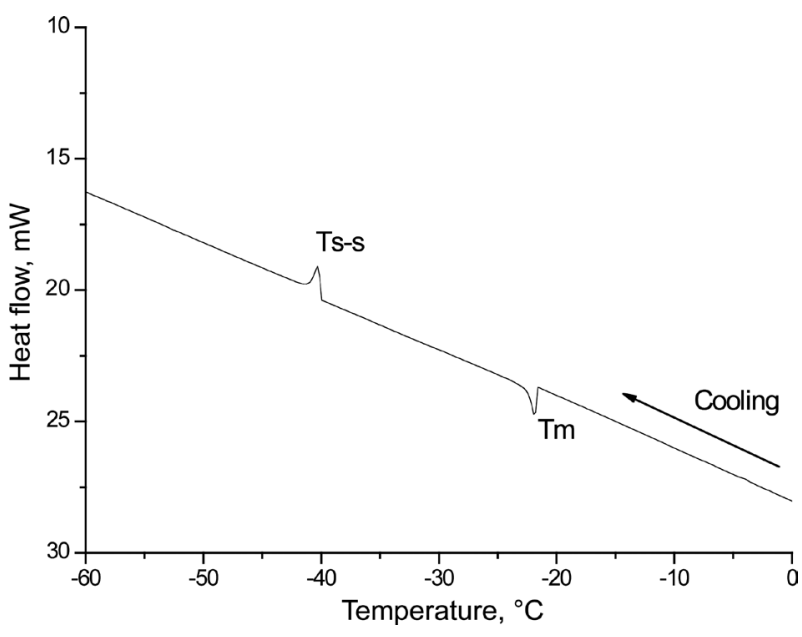

Fig. 5. DSC traces on second cooling for porphyrin 8

transition (Ts-s) before melting (compounds 5a, 8 and 9a). The third type of behavior is represented by the salt that only exhibited a glass transition at $200{ }^{\circ} \mathrm{C}$ without melting (5b). Figure 5 shows the DSC traces of one of the representatives, porphyrin $(\mathbf{8})$ as an example, Upon cooling, 8 undergoes a single liquid-solid phase transition at approximately $\mathrm{T}=-22^{\circ} \mathrm{C}$, showing an exothermic peak and exhibit afterwards a solid-solid phase transition at $-39^{\circ} \mathrm{C}$ with an endothermic peak.

A comparison of the melting point values of the asymmetric porphyrin based salts with those of the symmetric porphyrin based ones clearly evidences the influence of the asymmetry on the melting temperature. The melting point values of the asymmetric porphyrin salts (mono-alkylated) are lower than those for the corresponding symmetric salts (di-alkylated). For the same anion, the salts with lower symmetry porphyrins generally exhibit a lower melting point than those with higher symmetry (see Table 2, 5a vs. 9a, 5b vs. 9b, $\mathbf{7} v s . \mathbf{1 1}, \mathbf{6 b} v s . \mathbf{1 0}, \mathbf{8} v s . \mathbf{1 2}$ ). These results suggest that the packing efficiency of the salt could be disrupted by reducing the cation symmetry, thus lowering the melting point. It seems that the asymmetric factor and longer alkyl chains of the cationic porphyrin salt play a key role in achieving the low melting points of these salts.

The nature of the anion affects also significantly the melting point of the porphyrin salts. For these porphyrinbased salts, the influence of tetrakis(perfluorophenyl)borate groups on the melting point is remarkably obvious and tetrakis(perfluorophenyl)borate porphyrins $(\mathbf{6 a}, \mathbf{6 b}$, 8, 10 and 12) display melting temperatures that are greatly lower than that of bromide or iodide porphyrins (5a, 5b, 7, 9b and 11) (see Table 2). This is probably due to the effective charge distribution over the anion which tends to reduce the crystal lattice energy of the salts, thus resulting in low-melting salts [24].

\section{Conductivity}

Table 2 also shows the specific conductivity $(\kappa)$ of the three liquid porphyrin salts $(\mathbf{5 b}, \mathbf{6 b}$ and $\mathbf{8})$ at $22{ }^{\circ} \mathrm{C}$.
Indeed, we were not able to measure specific conductivity values for the other salts at higher temperatures. For the salts with the same cation, the specific conductivity is much higher for the $\mathrm{B}\left(\mathrm{C}_{6} \mathrm{~F}_{5}\right)_{4}^{-}(\mathbf{6 a}$ and $\mathbf{8})$ anion than $\mathrm{I}^{-}$anion $(\mathbf{5 b})$. For $\mathrm{B}\left(\mathrm{C}_{6} \mathrm{~F}_{5}\right)_{4}{ }^{-}$anion, the specific conductivity of $\mathbf{6 b}\left(4.01 \mathrm{mS} . \mathrm{cm}^{-1}\right)$ is greatly higher than $\mathbf{8}(0.05$ $\left.\mathrm{mS} . \mathrm{cm}^{-1}\right)$. This can be explained by the bigger cation size and molecular weight of the cation of 8 [24].

\section{CONCLUSION}

In summary, we have developed an effective synthetic method to prepare porphyrin derivatives bearing one pyridyl or two pyridyl groups by [2+2] aldehyde-dipyrromethane condensation in a one-pot reaction. Alkylation of the neutral porphyrins led to mono- or di-cationic porphyrin salts which were fully characterized by ${ }^{1} \mathrm{H}$ NMR, ${ }^{19} \mathrm{~F}$ NMR, MALDI-TOF mass spectrometry, elemental analysis and UV-visible spectroscopy. DSC studies also indicate that those new ionic liquid porphyrin exhibit relatively low melting points. Furthermore, $\mathbf{5 b}, \mathbf{6 b}$, and $\mathbf{8}$ salts are liquid at room temperature and their melting points are observed below $25{ }^{\circ} \mathrm{C}$. These results show that low symmetry of the cation and presence of large anions play an important role in decreasing melting points of these ionic liquid porphyrin derivatives. A specific conductivity value up to $4 \mathrm{mS} . \mathrm{cm}^{-1}$ was obtained for a compound $(6 \mathbf{b})$ having the coordinating anion $\mathrm{B}\left(\mathrm{C}_{6} \mathrm{~F}_{5}\right)_{4}^{-}$. Further work on complexation of these ILs is currently underway in our laboratory.

\section{Acknowledgements}

The Centre National de Recherche Scientifique (ICMUB, UMR CNRS 5260) is gratefully thanked for financial support. Hai-Jun Xu also gratefully acknowledges the "Région Bourgogne" and CNRS for a postdoctoral fellowship.

\section{Supporting information}

${ }^{1} \mathrm{H}$ and ${ }^{19} \mathrm{~F}$ NMR spectra, MALDI-TOF mass spectra and thermal analyses (Figs S1-S46) are given in the supplementary material. This material is available free of charge via the Internet at http://www.worldscinet.com/jpp/ jpp.shtml.

\section{REFERENCES}

1. Welton T. Coord. Chem. Rev. 2004; 248: 2459-2477.

2. Wilkes JS, Levisky JA, Wilson RA and Hussey CL. Inorg. Chem. 1982; 21: 1263-1264.

3. Welton T. Chem. Rev. 1999; 99: 2071-2084.

4. Wasserscheid P and Keim W. Angew. Chem. Int. Ed. 2000; 39: 3773-3789.

5. Seddon KR. J. Chem. Technol. Biotechnol. 1997; 68: $351-356$. 
6. Ngo HL, Le Compte K, Hargens L and McEwen AB. Thermochim. Acta. 2000; 357-358: 97-102.

7. Hagiwara R. Electrochemistry 2002; 70: 130-136.

8. Gjikaj M, Brockner W, Namysl J and Adam A. CrystEngComm. 2008; 10: 103-110.

9. Dieter KM, Dymek CJ, Heimer NE, Rovang JW and Wilkes JS. J. Am. Chem. Soc. 1988; 110: 2722-2726.

10. Zhan HY, Liu HY, Chen HJ and Jiang HF. Tetrahedron Lett. 2009; 50: 2196-2199.

11. Huddleston JG, Willauer HD, Swatloski RP, Visser AE and Rogers RD. Chem. Commun. 1998: 1765-1766.

12. Bosmann A, Datsevich L, Jess A, Lauter A, Schmitz C and Wasserscheid P. Chem. Commun. 2001: 24942495.

13. Ou ZP, Shen J, Shao JG, Wenbo E, Galezowski M, Gryko DT and Kadish KM. Inorg. Chem. 2007: 2775-2786.

14. Endres F and Abedin SZE. Phys. Chem. Chem. Phys. 2006; 8: 2101-2116.

15. Wang P, Zakeeruddin SM, Mozer JE and Grätzel M. J. Phys. Chem. B 2003; 107: 13280-13285.

16. Wang P, Zakeeruddin SM, Comte P, Exnar I and Grätzel M. J. Am. Chem. Soc. 2003; 125: 11661167.

17. Matsumoto H, Matsuda T, Tsuda T, Hagiwara R, Ito Y and Miyazaki Y. Chem. Lett. 2001: 26-27.

18. Garcia B, Lavallée S, Perron G, Michot C and Armand M. Electrochim. Acta 2004; 49: 45834588.

19. Yang SR, Jiang HF, Li YQ, Chen HJ and Xu YB. Tetrahedron 2008; 64: 2930-2937.

20. Dupont J, de Souza RF and Suqrez PAZ. Chem. Rev. 2002; 102: 3667-3692.

21. Carmichael AJ, Earle MJ, Holbery JD, Mc Cormac PB and Seddon KR. Org. Lett. 1999; 1: 997-1000.

22. Zhou ZB, Matsumoto H and Tatsumi K. Chem. Eur. $J$ 2006; 12: 2196-2212.

23. Zhou ZB, Matsumoto H and Tatsumi K. Chem. Eur. J. 2005; 11: 752-766.

24. Zhou ZB, Matsumoto H and Tatsumi K. Chem. Eur. J. 2004; 10: 6581-6591.

25. Reichert WM, Holbrey JD, Swatloski RP, Gutowski KE, Visser AE, Nieuwenhuyzen M, Seddon KR and Rogers RD. Cryst. Growth Des. 2007; 7: 1106-1114.
26. Egashira M, Yamamoto Y, Fukutake T, Yoshimoto N and Morita M. J. Fluorine Chem. 2006; 127: 12611264.

27. Langmaier J and Samec Z. Anal. Chem. 2009; 81: 6382-6389.

28. Takai A, Chkounda M, Eggenspiller A, Gros CP, Lachkar M, Barbe JM and Fukuzumi S. J. Am. Chem. Soc. 2010; 132: 4477-4489.

29. Barbe JM, Habermeyer B, Khoury T, Gros CP, Richard P, Chen P and Kadish KM. Inorg. Chem. 2010; 49: 8929-8940.

30. Ohta K, Nguyen-Tran H-D, Tauchi L, Kanai Y, Megumi T and Takagi Y. In Handbook of Porphyrin Science, Vol. 12, Kadish KM, Smith KM and Guilard R. (Eds.) World Scientific: New Jersey, 2011; pp 1-120.

31. Huang X, Zhu C, Zhang S, Li W, Guo Y, Zhan X, Liu Y and Bo Z. Macromolecules 2008; 41: 6895-6902.

32. Gust D, Moore TA and Moore AL. Acc. Chem. Res. 2009; 42: 1890-1898.

33. Liu Y, Zhang HJ, Lu Y, Cai YQ and Liu XL. Green Chem. 2007; 9: 1114-1119.

34. Hatay I, Su B, Méndez MA, Corminboeuf C, Khoury T, Gros CP, Bourdillon M, Meyer M, Barbe JM, Ersoz M, Zális S, Samec Z and Girault HH. J. Am. Chem. Soc. 2010; 132: 13733-13741.

35. Boyd PDW and Reed CA. Acc. Chem. Res. 2005; 38: $235-242$.

36. Ethirajan M, Chen YH, Joshi P and Pandey RK. Chem. Soc. Rev. 2011; 40: 340-362.

37. Aratani N, Kim D and Osuka A. Acc. Chem. Res. 2009; 42: 1922-1934.

38. Nowak-Krol A, Gryko D and Gryko DT. Chem. Asian J. 2010; 5: 904-909.

39. Maruyama S, Sato K and Iwahashi H. Chem. Lett. 2010: 714-716.

40. Romeo C, Sonia IM, Gianbattista P, Canavesi A, Michelucci E, Tiseni PS, Marzorati E, Checchia A, Giannone M, Jabes D, Romanò G, Brunati C, Candiani G and Castiglione F. J. Med. Chem. 2007; 50: 3077-3085.

41. Nakanishi T, Miyashita N, Michinobu T, WakayamaY, Tsuruoka T, Ariga K and Kurth DG. J. Am. Chem. Soc. 2006; 128: 6328-6329.

42. Ruzié C, Michaudet L and Boitrel B. Tetrahedron Lett. 2002; 43: 7423-7426. 